\title{
Influence of socio-economic indicators on electricity consumption of low voltage customers in Cameroon
}

\author{
Flora Isabelle Métégam Fotsing ${ }^{1,2}$, Donatien Njomo ${ }^{1}$, Réné Tchinda ${ }^{2}$ \\ ${ }^{1}$ Environmental Energy Technologies Laboratory (EETL), University of Yaounde I, PO Box 812, Yaounde, Cameroon \\ ${ }^{2}$ Laboratory of Industrial Systems and Environment of the University of Dschang, PO BOX 96, Dschang, Cameroon \\ Email address: \\ fotsing85@yahoo.fr (F. I. M. Fotsing),dnjomo@usa.net (D. Njomo), ttchinda@yahoo.fr (R. Tchinda)
}

\section{To cite this article:}

Flora Isabelle Métégam Fotsing, Donatien Njomo, Réné Tchinda. Influence of Socio-Economic Indicators on Electricity Consumption of Low Voltage Customers in Cameroon. International Journal of Energy and Power Engineering. Vol. 3, No. 4, 2014, pp. 186-203.

doi: $10.11648 /$ j.ijepe.20140304.13

\begin{abstract}
In this paper, the demand of Low Voltage electricity customers in Cameroon using electricity as an energy source beginning from the period 1975 to 2011 is modeled. This approach aims to study the consumption determinants (macro- economic indicators, demographic indicators and lagged consumption of low voltage electricity) of low Voltage Customers and to analyze those determinants that have a strong influence on consumption. Parameters estimated by EVIEWS 7.2 software for linear and exponential (CooB-Douglas) models were used. The results show that CooB-Douglass models are better than the linear model. It also shows that: (i) the best linear model is a function of delayed consumption $C_{t-1}$; overall gross domestic product $\left(\left(\mathrm{GDP}_{g}\right)_{t}\right)$ and population $\left(P_{t}\right)$; (ii) the best model CooB-Douglas is a function of delayed consumption $C_{t-1}$, the global gross domestic product $\left(\left(\mathrm{GDP}_{g}\right)_{t}\right)$ and the number of subscribers $\left(S_{t}\right)$. It noticed that the macroeconomic indicators have a better influence on demographic consumer's indicators and that the absence of the delayed consumption variable in a model causes autocorrelation of the residuals models.
\end{abstract}

Keywords: Consumption of Low Voltage Electricity, Linear Regression Models, Macro- Economic Indicators, CooB-Douglass Models, Socio-Economic Parameters, Demographic Indicators, Modeling

\section{Introduction}

A good supply of electricity contributes immensely to the development of national economy. Given its importance, it is vital for a country to ensure it accessibility and to guarantee it continuous supply, both in quantity and quality and at affordable prices. Giving that its nature those not permit it to be stored, the supply of energy must meet it demand at any time. Electricity demand as a direct reflection of the economic and social activity in a country, present a global predictable character, but with an uncertain margin. It is been influence in the long term by a number of factor amongst which we have socio-economic indicators as the principal influencing factor.

Despite the significant energy potential of the African continent, access to electricity remains scarce in many rural areas in Africa. Certain zones powered by electricity are prone to rationing with regular blackouts and low voltages in the network. In Cameroon, electricity supplied was enhanced by the American company AES-SONEL from
1994 to 2013 under the supervision of ARSEL (Electricity Sector Regulator Agency). Since 2014, electricity supply is been done by English company ACTIS. Despite the construction of a number of terminals to solve the deficiency of electricity supply in the country, the disequilibrium between the demand of electricity persist and this has resulted to blackouts and drops in voltage of electricity across the country. The cost generated by a power break down is enormous both for enterprises and the population and thus curbs socio- economic development of a country. It is therefore important to know the determinants of consumption so as to better manage available resources and advice the government on the development of energy policies.

The hydro- power generation accounts for the bulk of the domestic production with over $92 \%$. The share of thermal generation connected to the electricity network represents an average of $6.1 \%$ of the production of AES SONEL nation-wide during the period 2005 to 2011, with a maximum of $8.3 \%$ achieved in 2007. As for isolated power plants, they represent $1.6 \%$ of the national production of 
AES SONEL, with a maximum of $1.8 \%$ in 2011 [1]. Low voltage sales have been improving steadily with an increase of $32.9 \%$ in 2005 to $40.9 \%$ in 2011 [1]. $71 \%$ of low voltage customer in Cameroon is made up of households, $27 \%$ of professional institutions (universities, government, hotels ...) and $3 \%$ of public lighting. The domestic sector been the dominant consumer of low voltage (that is about $71 \%$ of the consumption), it is therefore important to determine the parameters that influence consumption in order to detect the model which the best suited to this analysis. Here public lighting has a very small percentage because a majority functions with integrated solar panel.

This work is organized into five sections: Section 2 provides a literature review. Section 3 describes the data used and devoted to the methodology of the analysis. Section 4 presents the results and section 5 presents the comments and conclusion.

\section{Literature Review}

Several studies have been conducted to explain the consumption of electricity in terms of socio -economic variables. Reference [12] analyzes the residential electric Appliance Holdings and Consumption. Reference [25] evaluates the cost and benefits of appliance efficiency standards. . Reference [6] model the electricity demand in the residential sector in Quebec for house heating, water and determine consumption in terms of economic variables. Reference [5] presents an integrated model for assessing the total energy demand in Quebec in the three economic sectors: residential, commercial and industrial sectors. Analysis shows the total energy demand as a function of its passed value, the real energy prices, real income and the degree of the days heating. Reference [24] proposes a model that permits to estimate the energy demand of Canadian residential customers following their energy consumption and household income. Reference [31] evaluates the effects of changing in electricity prices and the majeure consequences of such changes on the consumption of Californian households. Reference [17] shows that a unidirectional causal link from energy consumption to economic growth has been identified in the long and short term in the Mediterranean region.

However, very few studies have been conducted for the analysis of electricity consumption in emerging African country such as Cameroon which has different climate from other European, Asian or American countries. Reference [26] demonstrates in two steps Engle and Granger that there exist from the ADF test a cointegration between GDP and consumption of electrical energy in Mali. Reference [32] shows that using Granger's causality test, reveals the existence of a unidirectional causal link of the GDP on energy consumption in Congo. Reference [30] analyzes the impact of energy consumption on economic performance (overall GDP, primary GDP, secondary GDP and tertiary GDP) in Cameroon. He concluded that in the tertiary sector, energy consumption brings about an increase in production and the estimation that the first differentiation of VAR model shows that a $1 \%$ increase in the consumption of electrical energy leads to a $0.6 \%$ increase of the added value on services. Reference [37] shows that there is a strong positive correlation between the consumption of electrical energy and the level of economic activity in Ivory Coast, but for the fact that the relationship is unidirectional (the GDP explains the consumption of electricity and not the contrary). Reference [11] modeled the demand for electricity in Togo, taking into account in the region meteorological parameters only. He concludes that the results could be best if socio economic parameters were integrated.

\section{Data and Methodology of Modeling}

\subsection{Data}

In this paper, we are modeling the electrical consumption of low voltage electricity customers in Cameroon. To explain this consumption, we used three parameters namely lagged consumption, macroeconomic parameters (global GDP, tertiary GDP and GDP per capita) and demographic parameters (population, number of households, number of subscribers). Historical data is provided by AES-SONEL over the period 1975-2011. Macroeconomic and demographic data are provided by the National Institute of Statistics of Cameroon (INS). Reference [39] analyses the influence of consumption on economic growth

\subsubsection{Chronological Study}

We can distinguish three major periods of economic growth in Cameroon so far:

- $\quad$ The period 1960 - 1986: this period corresponded to the five year development plans, with sustainable growth and realizes global economic development goals, such as doubling the per capita income, attend at the beginning 1980s. This period is characterized by an explosion in the petroleum production sector (from 1977 to 1986), which resulted to a high increase of $8.7 \%$ in the GDP. It also notices an evolution in the Consumption of low voltage electricity at an average annual growth rate of $10.91 \%$ due to population growth and urbanization.

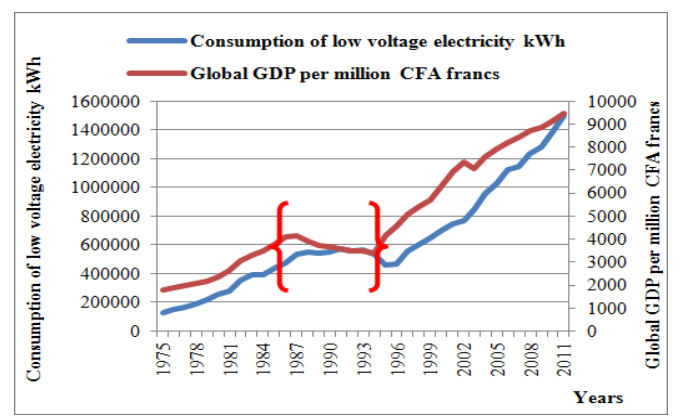

Figure 1. Evolution curves of low voltage electricity Consumption and overall GDP. 
- The period 1987 - 1996: this period was characterized by an economic crisis, resulting to higher interest rates, falling prices and an increase in the demand for importation of basics commodities, rising import prices, an increase of the external debt, a fall in the price of the U.S. dollar and the devaluation of the FCFA. The significant decrease in the overall income and the urgency to restore macro-economic equilibrium let to the suspension of the five-year development plan in favor of the structural adjustment programs spearheaded by international financial institutions. Thus, during this period of recession in which petroleum production witness drastic fell, the growth rate attained a negative average value of $4.1 \%$. This was accompanied by a decrease in the Consumption rate of low voltage electricity consumers with an average annual growth rate of $1.24 \%$. The devaluation of the CFA francs let to a decrease in the purchasing power of households.

- The period from 1996 to date: in spite of the fluctuation and the rapid downward trend in oil production during this period, it was characterized by a revival of economic growth and, this is thanks to the diversification of the national economy and the fact that there was less dependency on exportation in the petroleum sector. The real national GDP recorded an average annual growth rate of $3.9 \%$ over the period. In this regard, we note that the consumption of low voltage electricity has witness an average annual growth rate of $5.63 \%$ [2].

\subsubsection{Evolution of Macroeconomic Indicators and Consumption of Low Voltage Electricity}

Examination of the various graphs of Figure 2 suggests a consistency between the growth of consumption of low voltage electricity and different GDP. The determination coefficients between this consumption and the various GDP, that is, GDP/capita, the overall GDP and tertiary GDP are $0.7874,0.9293$ and 0.9422 respectively.

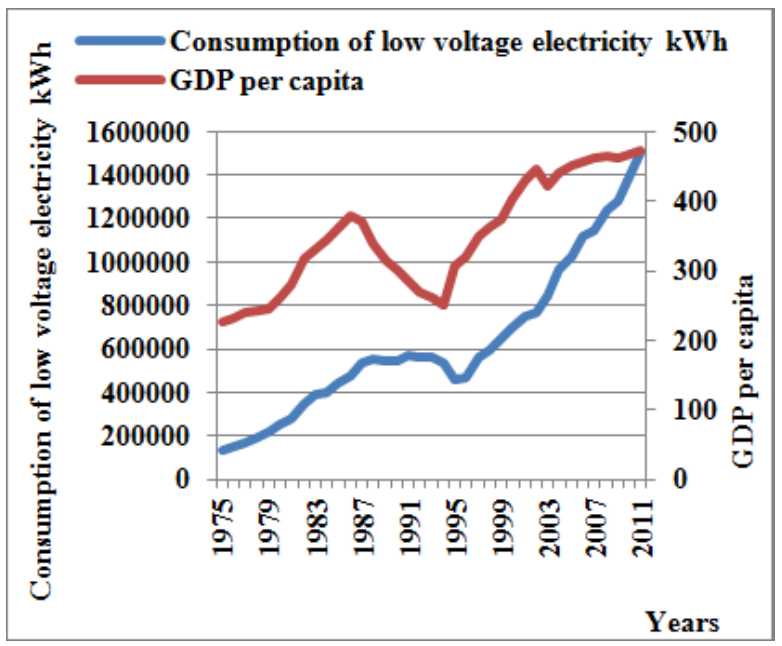

(a)

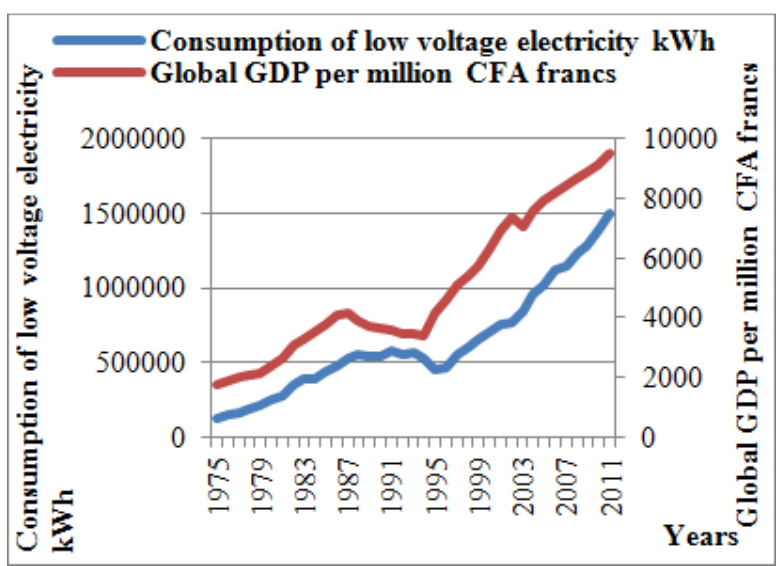

(b)

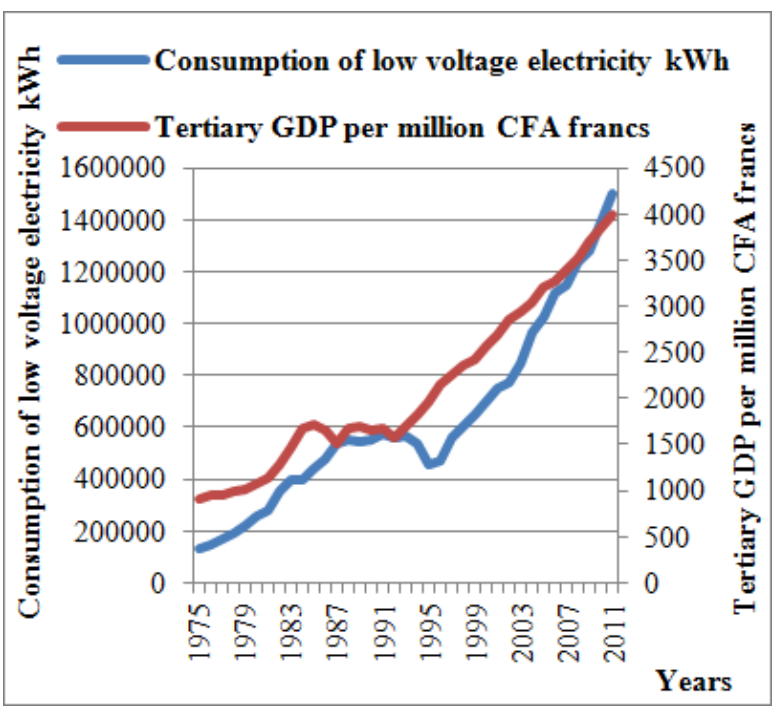

(c)

Figure 2. Evolution curves Consumption of low voltage electricity and various macroeconomic indicators (GDP/capita, global GDP and tertiary $G D P)$.

This enables us to confirm the positive impact that these different GDP can inflict on the consumption of low voltage electricity. We equally notice that consumption follows the evolution of GDP for all periods (the different GDPs evolve between 1960 to 1986 , decreased between 1987 to 1994, while consumption witness a slide increase and takes off again positively in 1995).The determination coefficient between consumption and tertiary GDP is higher and this can be justified by the fact that firms whose revenues constitute the GDP of the tertiary sector most often than not use Low Voltage electricity. These are professionals' low voltages (Hotels, Restaurants, universities, ministries ...) which cover about $27 \%$ of consumption. Therefore, different GDP have a positive influence on the Consumption of low voltage electricity. We equally notice that all variables increases with time. So they are not stationary. Since these variables depend on time, we can thus affirm that, we have time or chronological data. 


\subsubsection{Evolution of Demographic and Consumption of Low Voltage Electricity}

Examination of the various graphs of Figure 3 suggests a growth of all demographic indicators for all periods. The Consumption of low voltage electricity follows these growth trends except for the period 1987 - 1995, which was marked by the devaluation of the CFA francs. These caused a fall in the purchasing power of households and therefore let to a drastic drop in their consumption. The determining coefficients between consumption and various demographic indicators namely population, households and subscribers are $0.9023,0.9456$, and 0.9133 respectively. This allows us to confirm the positive impact that these indicators may have on the Consumption of low voltage electricity. Moreover, the determining coefficient between consumption and the number of households is higher; this is justified by the fact that the creation of a new household leads to additional power consumption.

Globally, we can observe over the entire history, a growing trend in the Consumption of low voltage electricity, which is proportional to the increase in population and the real per capita income, to minor fluctuations. This can be explained by the significant share of the households in the consumption of low voltage electricity, estimated at an averagely of $71 \%$ in recent years.

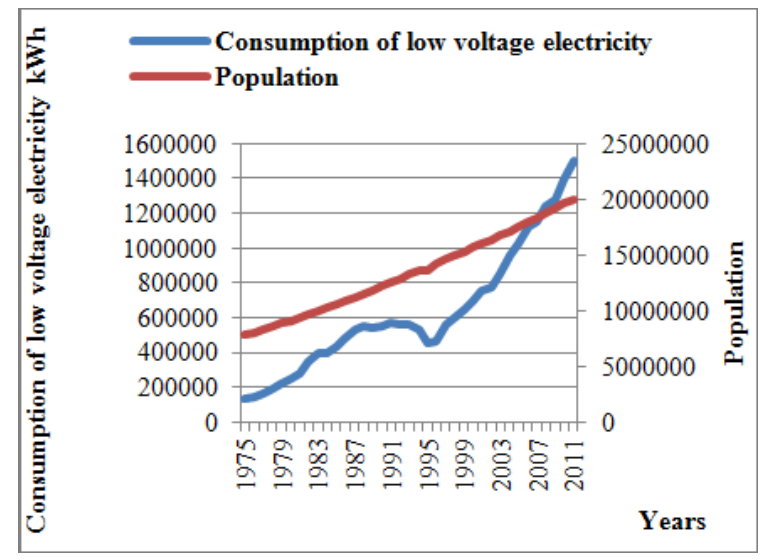

(a)

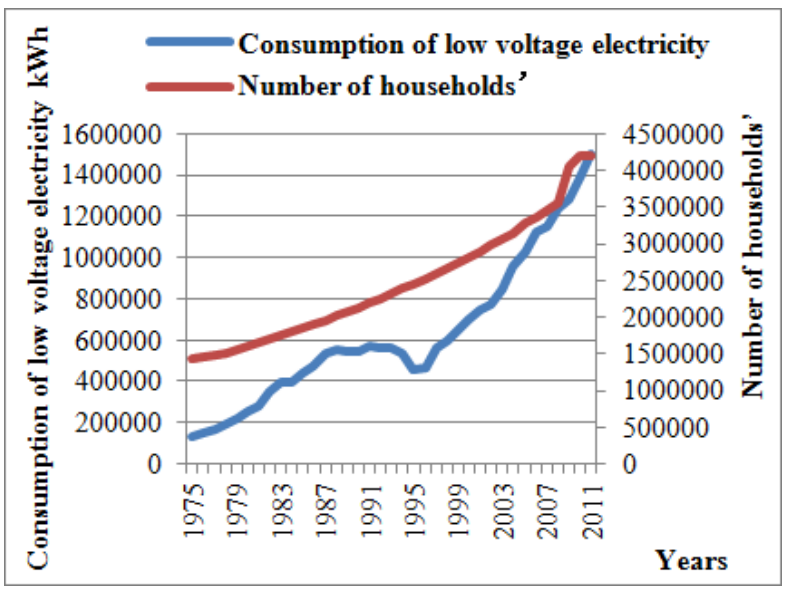

(b)

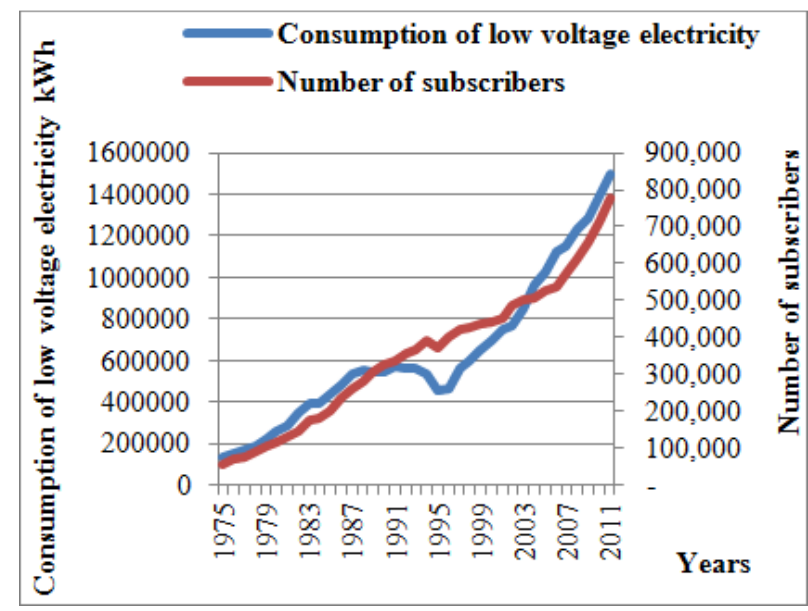

(c)

Figure 3. Evolution curves of the Consumption of low voltage electricity and various demographic indicators (population, households, and subscribers.

\subsection{Methodology of Modeling}

\subsubsection{Linear Models}

A time series is a set of observations that are distinguished by the important role that the order in which they were collected plays .The principal models for the study of time series are:

- $\quad$ Autoregressive models ("Auto - Regressive ").

They were introduced by Yule [34]. In these models, we take into account a linear dependence of the process of its own lag.

$$
X_{t}=\alpha_{1} X_{t-1}+\alpha_{2} X_{t-2}+\cdots+\alpha_{p} X_{t-p}+u_{t}
$$

Where $\mathrm{p} \in \mathrm{N} *$ is the order of the process $\alpha_{1}, \alpha_{2}, \ldots \alpha_{p}$ are real constants and $\left(u_{t}\right)_{\mathrm{ttZ}}$ is white noise

Moving average models («Moving Average») They were introduced by Slutsky [34]. A moving average process is the sum of white noise and its past:

$\operatorname{MA}(\mathrm{q}): X_{t}=u_{t}+\beta_{1} u_{t-1}+\beta_{2} u_{t-2}+\cdots+\beta_{p} u_{t-q}$

Where $\mathrm{q} \varepsilon \mathrm{N} *$ and $\beta_{1}, \beta_{2}, \ldots \beta_{p}$ are real constants .

- ARMA models ("Auto - Regressive Moving Average")Developed by Box \& Jenkins [34]. ARMA models are a combination of autoregressive and moving average models:

ARMA (p, q) : $X_{t}-\alpha_{1} X_{t-1}-\alpha_{2} X_{t-2}-\cdots-$ $\alpha_{p} X_{t-p}=u_{t}+\beta_{1} u_{t-1}+\beta_{2} u_{t-2}+\cdots+\beta_{p} u_{t-q}$

- The ARIMA (Autoregressive Integrated Moving Average) and SARIMA (Seasonal Autoregressive Integrated Moving Average) (a SARIMA process is an integrated ARMA process with seasonal component) [34]. ARIMA and SARIMA models were then developed in order to model a large number of real phenomena that present trends and / or seasonality. ARMA models are applied to integrated series.

Linear regression is a statistical technique used to model 
the linear relationship between exogenous variables (denoted $Y_{i t}$ ) and an endogenous variable (denoted by $X_{t}$ ).

$$
X_{t}=\alpha_{0}+\alpha_{1} X_{t-1}+\alpha_{2} Y_{1 t}+\alpha_{3} Y_{2 t}+\cdots+\alpha_{n} Y_{n t}+u_{t}
$$

Where:

$X_{t}$ is an endogenous variable which in our article stands for low voltage consumption.

$X_{t-1}$ is the lag value for the Consumption of low voltage electricity.

$Y_{i t}$ are the exogenous variables (global GDP , tertiary
GDP, population, households, number of subscribers ). $\alpha_{0}, \alpha_{1}, \ldots \alpha_{n}$ are the parameters to estimate from the model. $u_{t}$ is the white noise defined by the relation : $=\beta_{1} u_{t-1}+\beta_{2} u_{t-2}+\ldots+\beta_{P} u_{t-P}+\varepsilon_{t}$

The introduction of lag variables in our model permits to intercept the dynamic effects that are spread over several periods. Modeling consumption of low voltage electricity in Cameroon is expressed in terms of its lag value, macroeconomic and demographic variables: The linear models used are:

$$
\begin{gathered}
C_{t 1}=\alpha_{0}+\alpha_{1} C_{t-1}+\alpha_{2}(S)_{t}+\alpha_{3} H_{t}+\alpha_{4} P_{t}+\alpha_{5}\left(\frac{\mathrm{GDP}}{\text { Capia }}\right)_{t}+\alpha_{6}\left(\mathrm{GDP}_{g}\right)_{t}+\alpha_{7}\left(\mathrm{GDP}_{\text {tert }}\right)_{t}+u_{t} \\
C_{t 2}=\alpha_{0}+\alpha_{1} C_{t-1}+\alpha_{4} P_{t}+\alpha_{6}\left(\mathrm{GDP}_{g}\right)_{t}+u_{t} \\
C_{t 3}=\alpha_{0}+\alpha_{1} C_{t-1}+\alpha_{5}\left(\frac{\mathrm{GDP}}{\text { Capia }}\right)_{t}+\alpha_{6}\left(\mathrm{GDP}_{g}\right)_{t}+\alpha_{7}\left(\mathrm{GDP}_{\text {tert }}\right)_{t}+u_{t} \\
C_{t 4}=\alpha_{0}+\alpha_{1} C_{t-1}+\alpha_{2}(S)_{t}+\alpha_{3} H_{t}+\alpha_{4} P_{t}+u_{t} \\
C_{t 5}=\alpha_{0}+\alpha_{2}(S)_{t}+\alpha_{3} H_{t}+\alpha_{4} P_{t}+\alpha_{5}\left(\frac{\mathrm{GDP}}{\text { Capita }}\right)_{t}+\alpha_{6}\left(\mathrm{GDP}_{g}\right)_{t}+\alpha_{7}\left(\mathrm{GDP}_{\text {tert }}\right)_{t}+u_{t} \\
C_{t 6}=\alpha_{0}+\alpha_{5}\left(\frac{\mathrm{GDP}}{\text { Capita }}\right)_{t}+\alpha_{6}\left(\mathrm{GDP}_{g}\right)_{t}+\alpha_{7}\left(\mathrm{GDP}_{\text {tert }}\right)_{t}+u_{t} \\
C_{t 7}=\alpha_{0}+\alpha_{2}(S)_{t}+\alpha_{3} H_{t}+\alpha_{4} P_{t}+u_{t}
\end{gathered}
$$

Where

$C_{t}$ : Low voltage consumption in year $C_{t-1}$ : Lag Consumption of low voltage electricity

$\left(G D P_{g}\right)_{t}$ : Overall Gross domestic product (at constant prices) in year $\mathrm{t}$.

$\left(G D P_{\text {tert }}\right)_{t}$ : Tertiary Gross domestic product (at constant prices) in year $t$.

$\left(\frac{\text { GDP }}{\text { Capita }}\right)_{t}$ : Gross domestic product per capita (at constant prices) in year $t$.

$P_{t}$ : Population in year $\mathrm{t}(S)_{t}$ : Number of subscribers in year $\mathrm{t} H_{t}$ : Number of households in year $\mathrm{t}$.

Where $\alpha_{0}$ and $\left(\alpha_{1}, \alpha_{2}, \alpha_{3}, \alpha_{4}, \alpha_{5}, \alpha_{6}, \alpha_{7}\right)$ are intercept and regression coefficients, respectively. For handling serial correlation, we implement autoregressive in the error term of demand models. This usual method is expressed in (8) [34].Here, autoregressive order two model is applied in the composed regression models. As options, models without and with autoregressive order one model are computed as well.

$$
u_{t}=\beta_{1} u_{t-1}+\beta_{2} u_{t-2}+\ldots+\beta_{P} u_{t-P}+\varepsilon_{t}
$$

Where $u_{t}, \beta_{p}$ and $p$ are $\varepsilon_{t}$ error term, constant, autoregressive order, and a white noise, respectively.

\subsubsection{Models CooB-Douglas}

The general form of Coob-Douglas function is:

$$
Y=C \cdot \prod X_{i}^{a_{i}} \text { où } c, a_{i}>0
$$

Index $i$ corresponds to the factors of production. This form can be linearized by applying the logarithm:

$$
\ln (y)=\ln (c)+\sum_{i} a_{i} * \operatorname{Ln}\left(x_{i}\right)
$$

This particular form is used to calculate the elasticity of the average electricity demand directly.

Coob-Douglass' Models used are:

$$
\begin{gathered}
C_{t 1^{\prime}}=\mu_{0} *\left(C_{t-1}^{\mu_{1}}\right) *\left((S)_{t}^{\mu_{2}}\right) *\left(H_{t}^{\mu_{3}}\right) *\left(P_{t}^{\mu_{4}}\right) *\left(\left(\mathrm{GDP}_{g}\right)_{t}^{\mu_{6}}\right) *\left(\left(\mathrm{GDP}_{\text {tert }}\right)_{t}^{\mu_{7}}\right) * v_{t} \\
C_{t 2^{\prime}}=\mu_{0} *\left(C_{t-1}^{\mu_{1}}\right) *\left((S)_{t}^{\mu_{2}}\right) *\left(\left(\mathrm{GDP}_{g}\right)_{t}^{\mu_{6}}\right) * v_{t} \\
C_{t 3 \prime}=\mu_{0} *\left(C_{t-1}^{\mu_{1}}\right) *\left(\left(\frac{\mathrm{GDP}}{\text { Capita }}\right)_{t}^{\mu_{5}}\right) *\left(\left(\mathrm{GDP}_{g}\right)_{t}^{\mu_{6}}\right) *\left(\left(\mathrm{GDP}_{\text {tert }}\right)_{t}^{\mu_{7}}\right) * v_{t} \\
C_{t 4^{\prime}}=\mu_{0} *\left(C_{t-1}^{\mu_{1}}\right) *\left((S)_{t}^{\mu_{2}}\right) *\left(M_{t}^{\mu_{3}}\right) *\left(P_{t}^{\mu_{4}}\right) * v_{t} \\
C_{t 5 \prime}=\mu_{0} *\left((S)_{t}^{\mu_{2}}\right) *\left(H_{t}^{\mu_{3}}\right) *\left(P_{t}^{\mu_{4}}\right) *\left(\left(\mathrm{GDP}_{g}\right)_{t}^{\mu_{6}}\right) *\left(\left(\mathrm{GDP}_{\text {tert }}\right)_{t}^{\mu_{7}}\right) * v_{t} \\
C_{t 66^{\prime}}=\mu_{0} *\left(\left(\mathrm{GDP}_{g}\right)_{t}^{\mu_{6}}\right) *\left(\left(\mathrm{GDP}_{\text {tert }}\right)_{t}^{\mu_{7}}\right) *\left(\left(\frac{\mathrm{GDP}}{\text { Capita }}\right)_{t}^{\mu_{5}}\right) * v_{t}
\end{gathered}
$$




$$
C_{t 71}=\mu_{0}\left((S)_{t}^{\mu_{2}}\right) *\left(H_{t}^{\mu_{3}}\right) *\left(P_{t}^{\mu_{4}}\right) * v_{t}
$$

Where $\mu_{0}$ and $\left(\mu_{1}, \mu_{2}, \mu_{3}, \mu_{4}, \mu_{5}, \mu_{6}, \mu_{7}\right)$ are intercept and regression coefficients, respectively. For handling serial correlation, we implement autoregressive in the error term of demand models. This usual method is expressed in (8') [34].Here, autoregressive order two model is applied in the composed regression models. As options, models without and with autoregressive order one model are computed as well.

$$
v_{t}=\beta_{1} v_{t-1}+\beta_{2} v_{t-2}+\ldots+\beta_{P} v_{t-P}+\varepsilon_{t}
$$

Where $v_{t}, \beta_{p}, p$ and $\varepsilon_{t}$ are error term, constants, autoregressive order, and a white noise, respectively.

\section{Results}

We have used two models namely the linear model and the exponential model (which is also a linear model when applying the logarithm) in modeling low voltage electricity consumption.

\subsection{Linear Model}

In order to determine the most appropriate model for our study, we started by carrying out the Augmented DickeyFuller test (ADF) [14] and Phillips -Perron test [34] on the variables used to determine whether they are stationary. Having observed that no series is stationary, we integrated them in order to determine their stationary order. From these tests, we find that our variables are having a different stationary order.

\begin{tabular}{|c|c|c|c|c|c|c|c|c|c|}
\hline \multirow[t]{2}{*}{ variable } & \multicolumn{2}{|c|}{$\begin{array}{l}\text { Differentiation } \\
\text { of order } 0\end{array}$} & \multicolumn{2}{|c|}{$\begin{array}{l}\text { Differentiation } \\
\text { of order } 1\end{array}$} & \multicolumn{2}{|c|}{$\begin{array}{l}\text { Differentiation } \\
\text { of order } 2\end{array}$} & \multirow{2}{*}{$\begin{array}{l}\text { Differentiation } \\
\text { of order } 3 \\
\text { PP.test } \\
\text { p-value } \\
\end{array}$} & \multirow{2}{*}{$\begin{array}{l}\text { Result } \\
\text { ADF.test } \\
\text { p-value }\end{array}$} & \\
\hline & $\begin{array}{l}\text { PP.test } \\
\text { p-value }\end{array}$ & $\begin{array}{l}\text { ADF.test } \\
\text { p-value }\end{array}$ & $\begin{array}{l}\text { PP.test } \\
\text { p-value }\end{array}$ & $\begin{array}{l}\text { p-value } \\
\text { ADF.test }\end{array}$ & $\begin{array}{l}\text { PP.test } \\
\text { p-value }\end{array}$ & $\begin{array}{l}\text { ADF.test } \\
\text { p-value }\end{array}$ & & & \\
\hline$\left(\frac{G D P}{\text { Capita }}\right)_{t}$ & 0.7657 & 0.7147 & 0.0079 & 0.006 & $*$ & $*$ & $*$ & $*$ & $d=1$ \\
\hline$\left(G D P_{g}\right)_{t}$ & 0.9865 & 0.9933 & 0.0079 & 0.0079 & $*$ & $*$ & * & * & $d=1$ \\
\hline$\left.\left(G D P_{\text {tert }}\right)_{t}\right)$ & 0.9998 & 0.9999 & 0.0082 & 0.0084 & $*$ & $*$ & * & $*$ & $d=1$ \\
\hline$P$ & 1.0000 & 1.0000 & 0.0000 & 0.0000 & $*$ & $*$ & & $*$ & $d=1$ \\
\hline$H$ & 1.0000 & 0.8368 & 0.0008 & 1.0000 & 0.0001 & 0.9999 & 0.0000 & 0.0000 & $d=1$ \\
\hline$S$ & 0.9997 & 0.9998 & 0.0472 & 0.7145 & 0.0000 & 0.0000 & & & $d=2$ \\
\hline$C_{t}$ & 0.9999 & 1.0000 & 0.0529 & 0.0437 & 0.0000 & 0.0000 & * & $*$ & $d=2$ \\
\hline
\end{tabular}

Table 1. Augmented Dickey-Fuller (ADF) and Phillips-Perron (PP) tests for linear models

\begin{tabular}{|c|c|c|c|c|c|c|c|c|c|}
\hline \multirow{2}{*}{ variable } & \multicolumn{2}{|c|}{ Differentiation of order 0} & \multicolumn{2}{|c|}{ Differentiation of order 1} & \multicolumn{2}{|c|}{ Differentiation of order 2} & \multicolumn{2}{|c|}{$\begin{array}{l}\text { Differentiation of } \\
\text { order } 3\end{array}$} & \multirow[t]{2}{*}{ Résultats } \\
\hline & $\begin{array}{l}\text { PP.test } \\
\text { p-value }\end{array}$ & $\begin{array}{l}\text { ADF.test } \\
\text { p-value }\end{array}$ & $\begin{array}{l}\text { PP.test } \\
\text { p-value }\end{array}$ & $\begin{array}{l}\text { ADF.test } \\
\text { p-value }\end{array}$ & $\begin{array}{l}\text { PP.test } \\
\text { p-value }\end{array}$ & $\begin{array}{l}\text { ADF.test } \\
\text { p-value }\end{array}$ & $\begin{array}{l}\text { PP.test } \\
\text { p-value }\end{array}$ & $\begin{array}{l}\text { ADF.test } \\
\text { p-value }\end{array}$ & \\
\hline $\operatorname{Ln}\left(\left(\frac{G D P}{\text { Capita }}\right)_{t}\right)$ & 0.6305 & 0.5674 & 0.0070 & 0.0061 & $*$ & $*$ & $*$ & $*$ & $d=1$ \\
\hline $\operatorname{Ln}\left(\left(\boldsymbol{G} \boldsymbol{D} \boldsymbol{P}_{\boldsymbol{g}}\right)_{t}\right)$ & 0.7450 & 0.7245 & 0.0168 & 0.0168 & $*$ & $*$ & $*$ & $*$ & $\mathrm{~d}=1$ \\
\hline $\operatorname{Ln}\left(\left(\boldsymbol{G} \boldsymbol{D} \boldsymbol{P}_{\text {tert }}\right)_{t}\right)$ & 0.8879 & 0.8947 & 0.0036 & 0.0027 & $*$ & $*$ & $*$ & $*$ & $d=1$ \\
\hline $\operatorname{Ln}(P)$ & 0.0017 & 0.0000 & $*$ & * & $*$ & * & $*$ & $*$ & $d=0$ \\
\hline $\operatorname{Ln}(H)$ & 1.0000 & 0.9979 & 0.0000 & 0.9688 & 0.0000 & 0.9999 & 0.0000 & 0.0000 & $d=1$ \\
\hline $\operatorname{Ln}(S)$ & 0.0002 & 0.0000 & $*$ & * & $*$ & $*$ & $*$ & $*$ & $d=0$ \\
\hline $\operatorname{Ln}\left(C_{t}\right)$ & 0.3529 & 0.5823 & 0.0153 & 0.0154 & * & * & * & * & $d=1$ \\
\hline
\end{tabular}

Table 2. Augmented Dickey-Fuller (ADF) and Phillips-Perron (PP) tests for Coob-Douglass models

Macroeconomic and demographics variables are integrated of order 1. $C_{t}$ is integrated of order 2. Results of these tests are included in Table 1. Now, we can apply the time linear regression because the theoretical conditions are satisfied.

In order to determine the most appropriate variables for our study, we first wrote a linear equation where all the variables were involved, we then analyzed their different coefficients using Student's test, whose results are listed in Table 3. The threshold of $\alpha=5 \%$, the law on Student's table gives us a critical value of 2.0452 at 29 degrees of freedom.

It is clear from this analysis that only the coefficients of $C_{t-1}, P_{t}$ and $\left(\mathrm{GDP}_{g}\right)_{t}$ have a higher magnitude than this critical value. Schwartz criterion (SC) and Akaike (AIC) having declined and the determination coefficient $\left(\mathrm{R}^{2}\right)$ having increase, during the withdrawal of other variables $\left(H_{t},(S)_{t},\left(\mathrm{GDP}_{\text {tert }}\right)_{t}\right.$ in the model. we can conclude that only three variables identified above play an explanatory role in the model. 
Table 3. Regression coefficients for linear models

\begin{tabular}{|c|c|c|c|c|c|c|c|c|}
\hline \multirow{2}{*}{$\begin{array}{l}\text { Explanation } \\
\text { Variable }\end{array}$} & \multicolumn{2}{|l|}{ Model 1} & \multicolumn{2}{|l|}{ Model 2} & \multicolumn{2}{|l|}{ Model 3} & \multicolumn{2}{|l|}{ Model 4} \\
\hline & Coef. & Prob.(p-value) & Coef. & Prob.(p-value) & Coef. & Prob.(p-value) & Coef. & Prob.(p-value) \\
\hline \multirow{2}{*}{$\mathrm{C}_{\mathrm{t}-1}$} & 0.8451 & 0 & 0.9407 & 0 & 0.9339 & 0 & 0.9992 & 0 \\
\hline & -10.563 & $0.0800 *$ & -17.95 & $0.0524^{*}$ & -15.026 & $0.0621^{*}$ & -12.94 & $0.0772 *$ \\
\hline \multirow{2}{*}{$(\mathrm{S})_{\mathrm{t}}$} & 0.4382 & 0.2202 & & & & & -0.4442 & 0.0672 \\
\hline & -1.2539 & $0.3494 *$ & & & & & $(-1.896)$ & $0.2342 *$ \\
\hline \multirow{2}{*}{$\mathrm{H}_{\mathrm{t}}$} & -0.0079 & 0.9293 & & & & & 0.1034 & 0.0795 \\
\hline & $(-0.089)$ & $0.0890 *$ & & & & & -1.8129 & $0.0570 *$ \\
\hline \multirow{2}{*}{$P_{t}$} & -0.0427 & 0.0387 & -0.0135 & 0.0242 & & & 0.006 & 0.6381 \\
\hline & $(-2.169)$ & $0.0197^{*}$ & $(-2.366)$ & $0.0057^{*}$ & & & -0.475 & $0.0126^{*}$ \\
\hline \multirow{2}{*}{$\left(\frac{\mathrm{GDP}}{\text { Capita }}\right)_{\mathrm{t}}$} & -513.08 & 0.3223 & & & 74.6656 & 0.7967 & & \\
\hline & $(-1.007)$ & $509.24 *$ & & & -0.2598 & $287.34 *$ & & \\
\hline \multirow{2}{*}{$\left(\mathrm{GDP}_{\text {glob }}\right)_{\mathrm{t}}$} & 91.1151 & 0.0429 & 38.5904 & 0.0001 & 38.5267 & 0.198 & & \\
\hline & -2.1213 & $42.951 *$ & -4.011 & $8.9687^{*}$ & $(-0.906)$ & $29.290 *$ & & \\
\hline \multirow{2}{*}{$\left(\mathrm{GDP}_{\text {tert }}\right)_{\mathrm{t}}$} & -26.408 & 0.7231 & & & -56.72 & 0.3718 & & \\
\hline & $(-0.357)$ & $73.797^{*}$ & & & $(-0.906)$ & $62.585^{*}$ & & \\
\hline \multirow{2}{*}{$\alpha_{0}$} & 353745 & 0.1669 & 63852.8 & 0.1058 & -20046 & 0.7593 & -140058 & 0.0671 \\
\hline & -1.4189 & $249299 *$ & -1.664 & 38367.* & $(-0.309)$ & 64852.* & $(-1.897)$ & $73796^{*}$ \\
\hline
\end{tabular}

Table 3. continued

\begin{tabular}{|c|c|c|c|c|c|c|}
\hline \multirow{2}{*}{$\begin{array}{l}\text { Explanation } \\
\text { Variable }\end{array}$} & \multicolumn{2}{|l|}{ Model 5} & \multicolumn{2}{|l|}{ Model 6} & \multicolumn{2}{|l|}{ Model 7} \\
\hline & Coef. & Prob.(p-value) & Coef. & Prob.(p-value) & Coef. & Prob.(p-value) \\
\hline \multicolumn{7}{|l|}{$\mathrm{C}_{\mathrm{t}-1}$} \\
\hline \multirow{2}{*}{$(\mathrm{S})_{\mathrm{t}}$} & 2.4587 & 0.0006 & & & 0.4744 & 0.3951 \\
\hline & -3.8471 & $0.6391 *$ & & & -0.8617 & $0.5505^{*}$ \\
\hline \multirow{2}{*}{$\mathrm{H}_{\mathrm{t}}$} & 0.01204 & 0.9511 & & & 0.521 & 0 \\
\hline & -0.0618 & $0.1946^{*}$ & & & -4.7277 & $0.1102 *$ \\
\hline \multirow{2}{*}{$\mathrm{P}_{\mathrm{t}}$} & -0.1525 & 0.0001 & & & -0.0428 & 0.1591 \\
\hline & $(-4.345)$ & $0.0351 *$ & & & $(-1.440)$ & $0.0297 *$ \\
\hline \multirow{2}{*}{$\left(\frac{\mathrm{GDP}}{\text { Capita }}\right)_{\mathrm{t}}$} & -1478.5 & 0.1645 & -805.95 & 0.3049 & & \\
\hline & $(-1.425)$ & $1037.5^{*}$ & $(-1.042)$ & $773.29 *$ & & \\
\hline \multirow{2}{*}{$\left(\mathrm{GDP}_{\text {glob }}\right)_{\mathrm{t}}$} & 232.274 & 0.0114 & 76.0399 & 0.356 & & \\
\hline & -2.6967 & $86.130 *$ & -0.936 & $81.223 *$ & & \\
\hline \multirow{2}{*}{$\left(\mathrm{GDP}_{\text {tert }}\right)_{\mathrm{t}}$} & -0.9116 & 0.9955 & 252.332 & 0.1374 & & \\
\hline & $(-0.005)$ & $161.14^{*}$ & -1.522 & $165.75^{*}$ & & \\
\hline \multirow{2}{*}{$\alpha_{0}$} & 1150369 & 0.0277 & 1864.7 & 0.9917 & -244284 & 0.1877 \\
\hline & -2.3145 & $497006^{*}$ & -0.01 & $177233^{*}$ & $(-1.345)$ & $181597 *$ \\
\hline
\end{tabular}

() t-statistic, *adjs. standard error, the related variable is not significant

We equally notice that the coefficient of $C_{t-1}$ is the most significant in the model $(t=10563>>2.02452)$.

This indicates the presence of a dynamic adjustment of $C_{t}$ in this model. The best model been based on the smallest values of AIC and SC, we can conclude that the most appropriate model for our study is that which depends on the three explanatory variables namely $P_{t},\left(\mathrm{GDP}_{g}\right)_{t}$ and $C_{t-1}$. It corresponds to the model 2 and the corresponding equation is $C_{t 2}$.

We observe that some parameters of different indicators have a great influence on consumption than others, but this does not mean that the other variables do not influence consumption. It may simply mean that, put together these parameters affect less consumption. In models 6 and 7 where consumption depends respectively on macro economic and demographic parameters, the coefficients of $\left(\mathrm{GDP}_{\text {tert }}\right)_{t}\left(\frac{\mathrm{GDP}}{\text { Capita }}\right)_{t}$ are more significant than the $\left(\mathrm{GDP}_{g}\right)_{t}$ in the first and those of $H_{t}$ and $(S)_{t}$ are more significant than that of $P_{t}$ in the second contrary to our expectations. The result of regression coefficients for linear models is listed in Table 3.

We can now perform Fischer's test (F-stat) which is a 
global significance test of coefficients. Threshold of $\alpha=5 \%$, $\mathrm{F} *(1,29)=249.8$.

There is overall significance of coefficients if $\mathrm{F} *(1,29)$ $<\mathrm{F}$. For 5, 6 and 7 models, $(\mathrm{F}<\mathrm{F} *)$. This is due to the fact that in these different models, consumption does not depend on the past value $C_{t-1}$. This is detrimental however; the introduction of past variables in this model would have permitted the intercept of the dynamic effects that are spread over several periods.

Table 4. Regression statistics of linear models

\begin{tabular}{|c|c|c|c|c|c|c|c|c|c|c|c|c|}
\hline \multirow{2}{*}{$\begin{array}{l}\text { Linear } \\
\text { Models }\end{array}$} & \multirow[b]{2}{*}{$\mathbf{R}^{2}$} & \multirow[b]{2}{*}{$\mathbf{R}^{2^{\prime}}$} & \multirow[b]{2}{*}{ SE Reg. } & \multirow{2}{*}{$\begin{array}{l}\text { Prob. } \\
\text { (F-Sta) }\end{array}$} & \multirow[b]{2}{*}{ AIC } & \multirow[b]{2}{*}{ SC } & \multicolumn{2}{|l|}{ White-test } & \multirow{2}{*}{$\begin{array}{l}\text { JB-test } \\
\text { (p-value) }\end{array}$} & \multirow[b]{2}{*}{ F -Stat } & \multicolumn{2}{|c|}{ B-D (LM) D-W } \\
\hline & & & & & & & $\begin{array}{l}\text { ORS } \\
\text { (p-value) }\end{array}$ & $\begin{array}{l}\text { F-stat } \\
\text { (p-value) }\end{array}$ & & & $\begin{array}{l}\text { LM } \\
\text { (p-value) }\end{array}$ & $\begin{array}{l}\text { F- stat } \\
\text { (p-value) }\end{array}$ \\
\hline $\begin{array}{l}\text { Model } 1 \\
\left(\boldsymbol{C}_{\boldsymbol{t} \mathbf{1}}\right)\end{array}$ & 0.9943 & 0.9929 & 29815.54 & 0.0000 & 23.63658 & 23.98847 & $\begin{array}{l}35.8860 \\
0.3801\end{array}$ & $\begin{array}{l}9.2607 \\
0.2555\end{array}$ & $\begin{array}{l}7.8431 \\
(0.0198)\end{array}$ & 704.9302 & $\begin{array}{l}9.870465 \\
(0.0427)\end{array}$ & $\begin{array}{l}2.2665 \\
(0.0917)\end{array}$ \\
\hline $\begin{array}{l}\text { Model } 2 \\
\left(\boldsymbol{C}_{\boldsymbol{t} 2}\right)\end{array}$ & 0.9937 & 0.9931 & 29448.27 & 0.0000 & 23.5231 & 23.6990 & $\begin{array}{l}9.2879 \\
(0.4111)\end{array}$ & $\begin{array}{l}1.0044 \\
0.4612\end{array}$ & $\begin{array}{l}5.9721 \\
(0.0504)\end{array}$ & 1685.021 & $\begin{array}{l}8.7714 \\
(0.0671)\end{array}$ & $\begin{array}{l}2.25499 \\
(0.0884)\end{array}$ \\
\hline $\begin{array}{l}\text { Model } 3 \\
\left(\boldsymbol{C}_{t 3}\right)\end{array}$ & 0.992954 & 0.9920 & 31664.72 & 0.0000 & 23.6920 & 23.9119 & $\begin{array}{l}16.0706 \\
(0.3091)\end{array}$ & $\begin{array}{l}1.2095 \\
(0.3375)\end{array}$ & $\begin{array}{l}21.6423 \\
\left(2 * 10^{\mathrm{E}}-5\right)\end{array}$ & 1092.206 & $\begin{array}{l}1.559394 \\
(0.8161)\end{array}$ & $\begin{array}{l}0.305625 \\
(0.8716)\end{array}$ \\
\hline $\begin{array}{l}\text { Model } 4 \\
\left(\boldsymbol{C}_{\boldsymbol{t} 4}\right)\end{array}$ & 0.991705 & 0.9906 & 34358.31 & 0.0000 & 23.85532 & 24.07525 & $\begin{array}{l}7.9173 \\
(0.4416)\end{array}$ & $\begin{array}{l}0.9515 \\
(0.4926)\end{array}$ & $\begin{array}{l}11.2780 \\
(0.0035)\end{array}$ & 926.5003 & $\begin{array}{l}2.091340 \\
(0.7190)\end{array}$ & $\begin{array}{l}0.416311 \\
(0.7954)\end{array}$ \\
\hline $\begin{array}{l}\text { Model } 5 \\
\left(\boldsymbol{C}_{\boldsymbol{t} 5}\right)\end{array}$ & 0.9724 & 0.9669 & 65504.28 & 0.0000 & 25.18628 & 25.4910 & & & & 176.3218 & {$[0.5408]$} & \\
\hline $\begin{array}{l}\text { Model } 6 \\
\left(\boldsymbol{C}_{\boldsymbol{t} \mathbf{6}}\right)\end{array}$ & 0.9441 & 0.9390 & 88913.42 & 0.0000 & 25.73052 & 25.90467 & & & & 185.8270 & {$[0.2439]$} & \\
\hline $\begin{array}{l}\text { Model } 7 \\
\left(\boldsymbol{C}_{\boldsymbol{t} 7}\right)\end{array}$ & 0.9488 & 0.9442 & 85051.21 & 0.0000 & 25.6417 & 25.8158 & & & & 204.1088 & {$[0.4198]$} & \\
\hline
\end{tabular}

() p-value, [ ] Durbin-Watson-stat.

In order to verify the absence of autocorrelation of residuals, we performed the Breusch-Godfrey test (BG) [20] on models $1,2,3$ and 4 , because in these models we have lag endogenous variables $\left(C_{t-1}\right)$ among exogenous variables. The Durbin-Watson test (DW) [34] shall be solely applied for models 5, 6 and 7 because there are no endogenous variables in the model.

Residuals are correlated at threshold $\alpha=5 \%$ if P-value $<$ $5 \%$. BG analysis test at order 4 and threshold $\alpha=5 \%$, shows that $\mathrm{P}$-value $>0.05$ for model 1,3 and 4 . $\mathrm{P}$-value $=$ $0.0427<0.05$ for model 2 . meanwhile, the analysis of square residue correlograms permits us to say that there is no autocorrelation of residues, however, the critical probability of Ljung-Box Statistics (L-B) [34] is for all delays superior at threshold $\alpha=5 \%$. We can therefore accept the hypothesis of an absence of autocorrelation between residues for model 1, 2, 3 and 4. The Analysis of the squared residue correlograms, White's test (1980) and ARCH LM's test [15] applied to these four models permits us to accept the hypothesis of an absence of heteroscedasticity errors given that at the threshold $\alpha=5 \%$, $\mathrm{P}$-value $>0.05$.

Our residue being homoscedastic it is thus white noise. DW test permits us to accept the hypothesis of an autocorrelation of residues, as models 5, 6 and 7 this test return values that are below 1.96 which is the critical value at the threshold $\alpha=5 \%$. For these models the autocorrelation can be rectified by introducing an autoregressive term. The results of these tests are listed in Table 2-b.

In order to verify if our white noise is Gaussian, we have performed Jarque -Bera's test (JB) [34]. The hypothesis of obtaining a Gaussian white noise is true at threshold $\alpha=$
$5 \%$, if Jarque -Bera's statistic is greater than that read from chi-square's table (JB stat $<5.99$ ) and the probability of Jarque -Bera's statistic provided by Eviews is superior to the provided threshold ( $\mathrm{P}$-value $>5 \%$ ).

The analysis of this test permits us to reject the hypothesis of a Gaussian white noise for all models except for Model 2. This model fulfill the conditions of Gaussian white noise namely $(\mathrm{JB}$ stat $=5.97<599)$ and $(\mathrm{P}$-value $=$ $0.0504>0.05)$. The CUSUM test [9] permits us to affirm the stability of our different models given that the curves are within the confident range at the threshold $\alpha=5 \%$. The results of the various tests are listed in Table 2-b.

For linear model, we have the following graphs: (A) forecast series in blue and forecast intervals to $95 \%$ in red. (B) Observation series in red, forecast series in green and residue in blue);

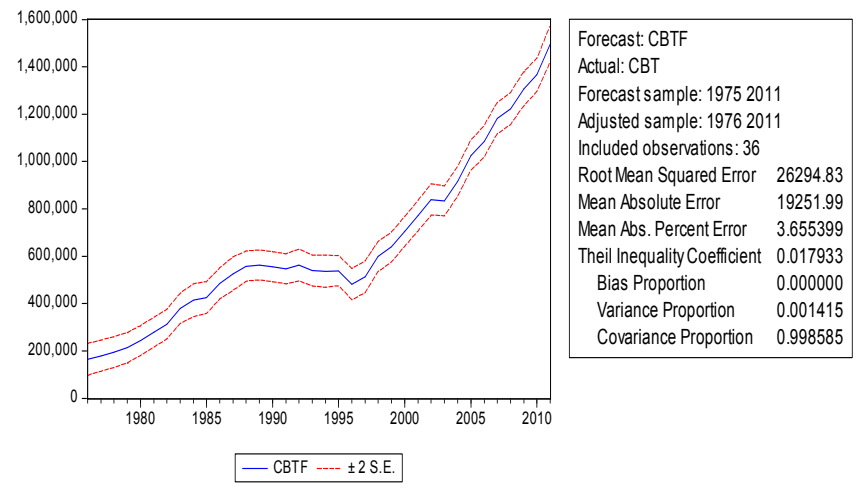

(A) 


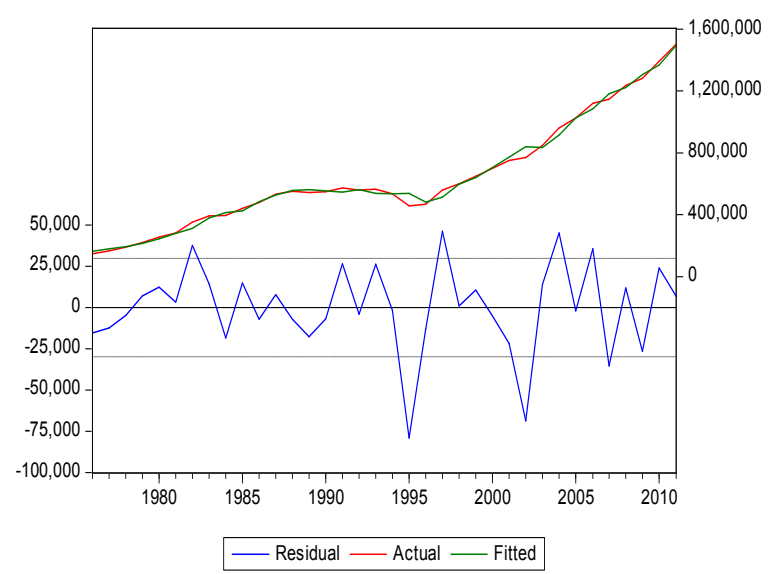

(B)

Figure 3-1. model 1

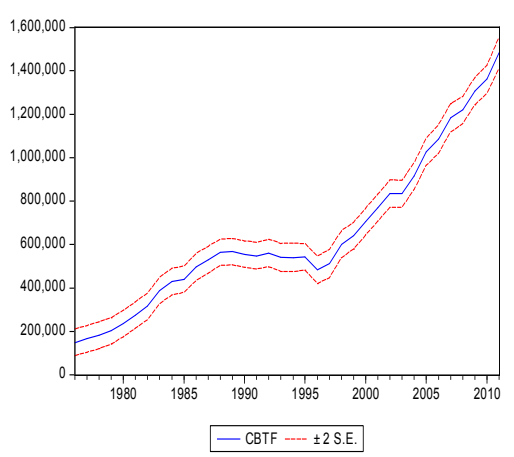

(A)

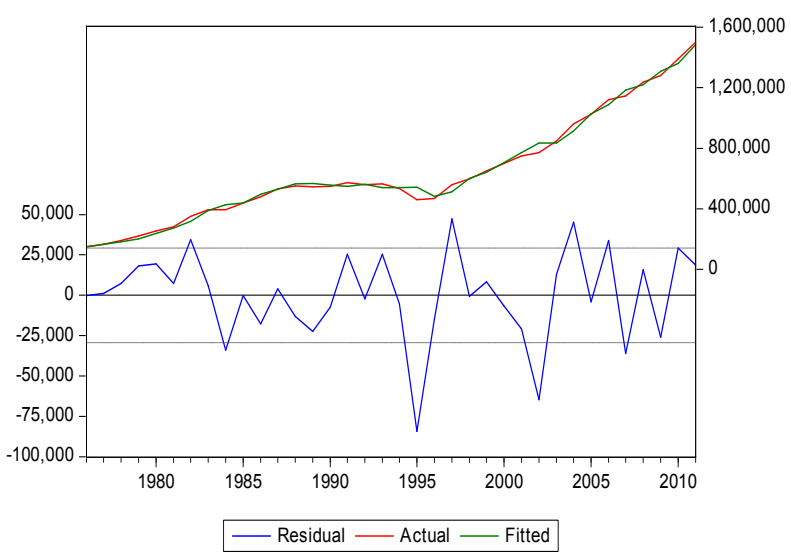

(B)

Figure 3-2. model 2
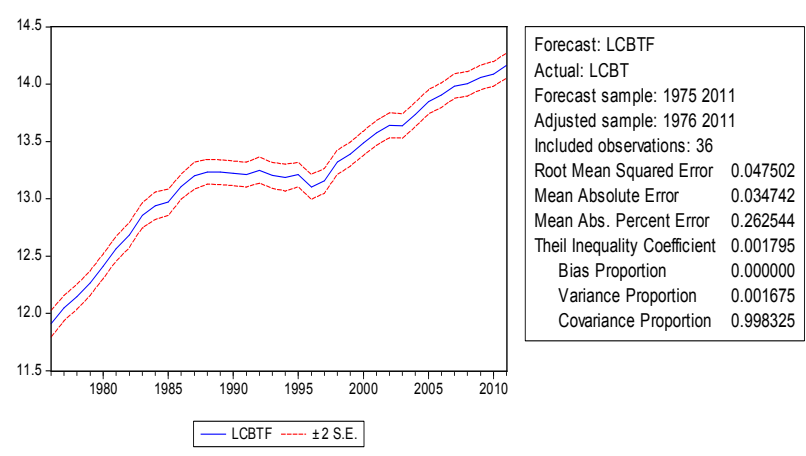

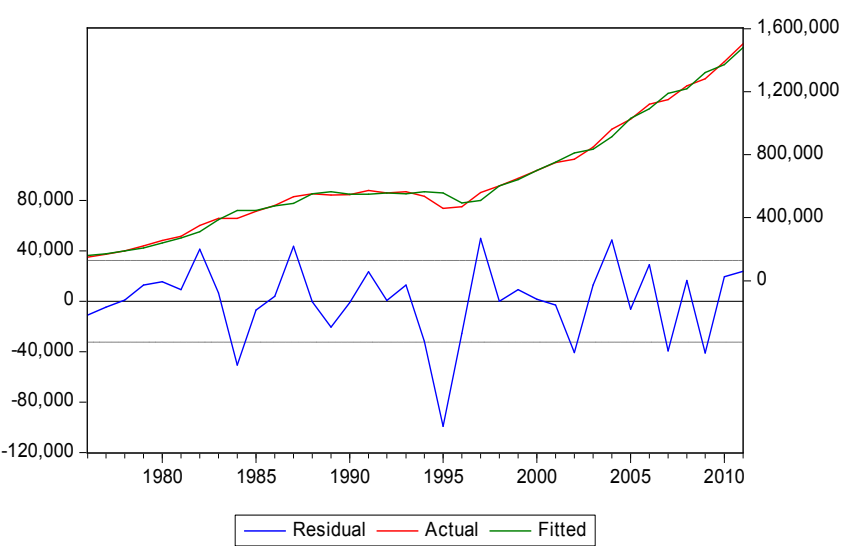

(B)

Figure 3-3. model 3
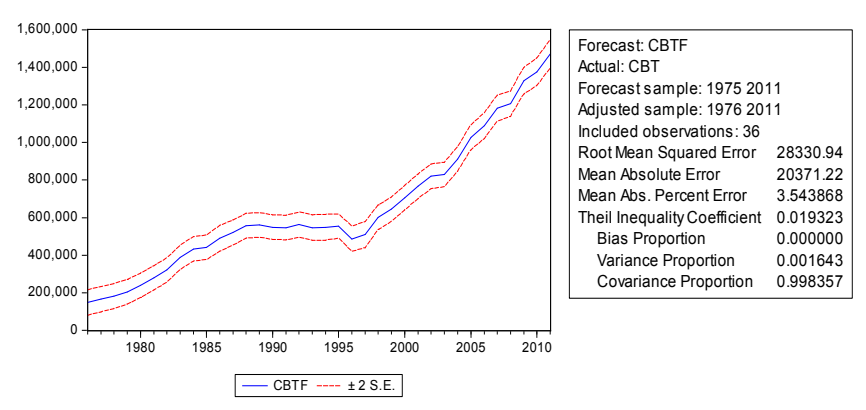

(A)

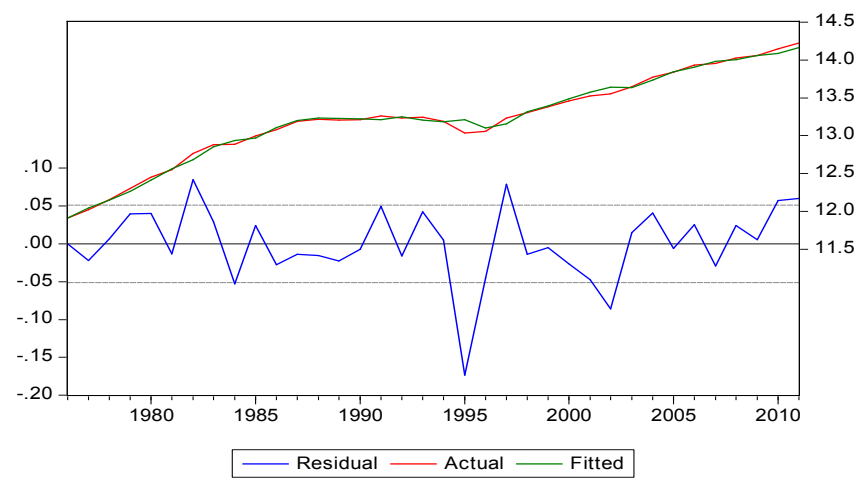

(B)

Figure 3-4. model 4

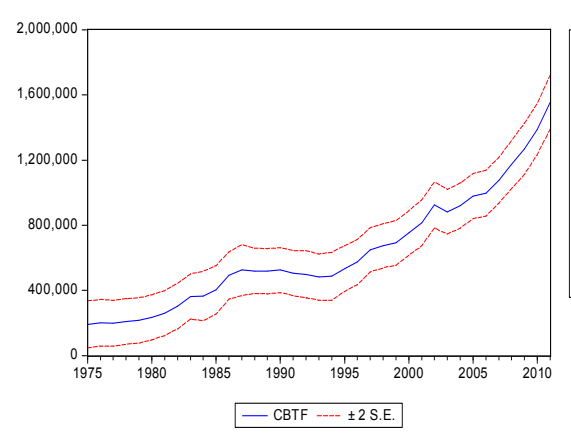

Forecast: CBT

Actual: CBT
Forecast sample: 19752011

Forecast sample: 1975201

Included observations: 37
Root Mean Squared Error $\quad 58983.35$

Mean Absolute Error $\quad 49319.84$

Mean Abs. Percent Error $\quad 10.27457$

Theil Inequality Coefficient 0.040819

Bias Proportion $\quad 0.000000$

$\begin{array}{ll}\text { Variance Proportion } & 0.006991 \\ \text { Covariance Proportion } & 0.993009\end{array}$ 


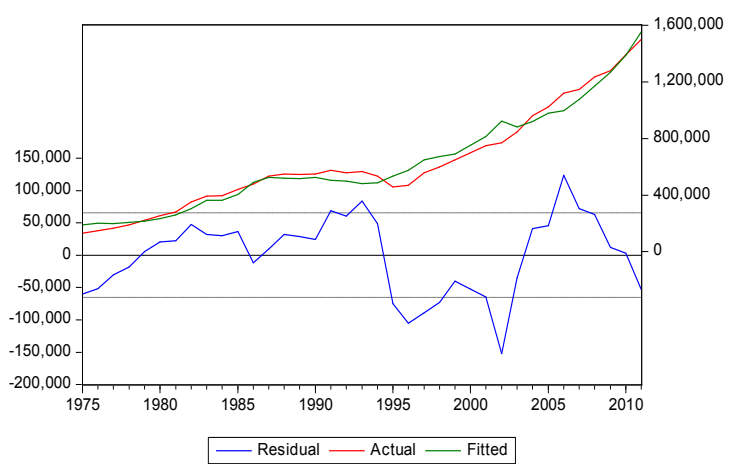

(B)

Figure 3-5. model 5

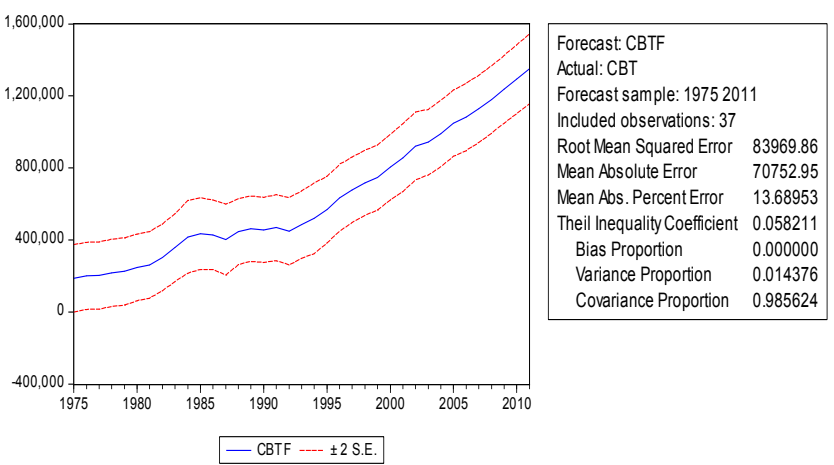

(A)

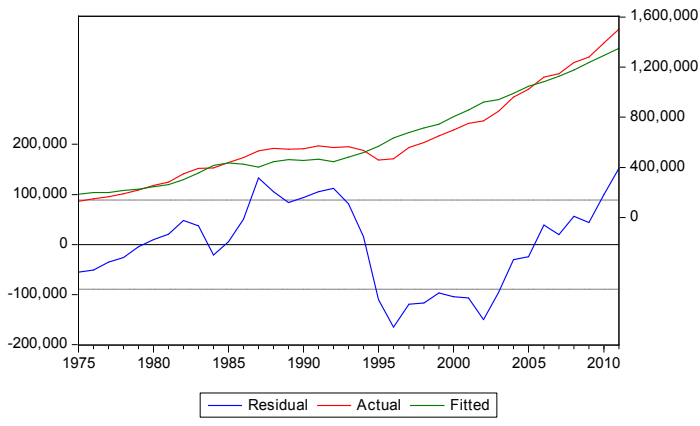

(B)

Figure 3-6. model 6

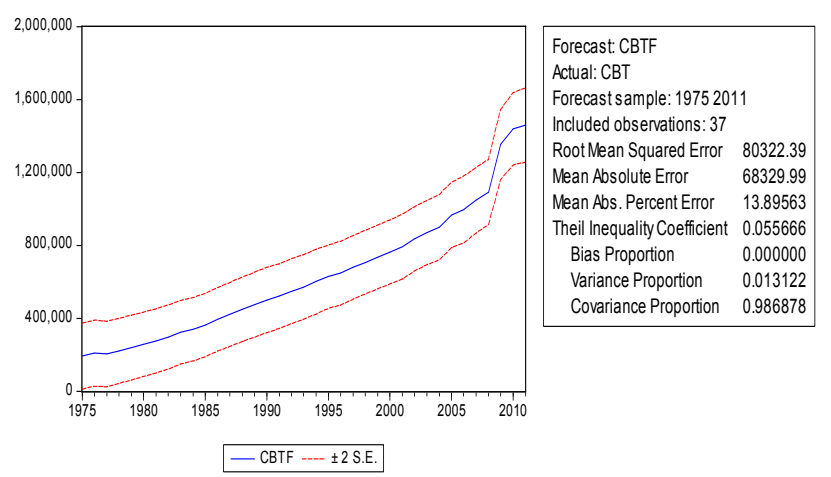

(A)

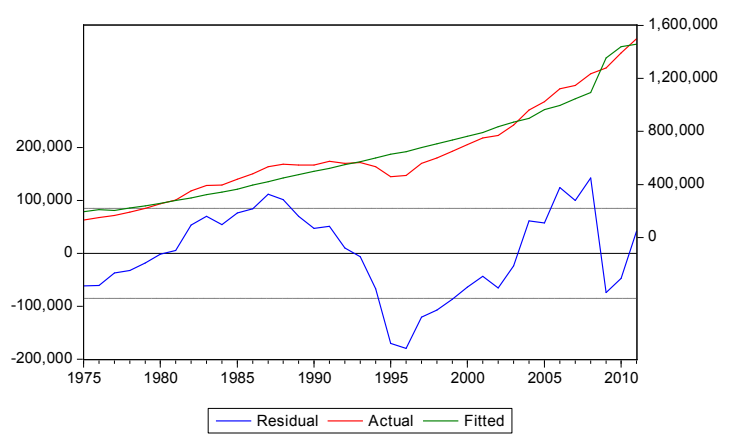

(B)

Figure 3-7. model 7

From the analysis of the different models, we can conclude that macro-economic indicators have a better influence on consumption than demographic indicators because SC and AIC are higher in Model $4\left(C_{t 4}=\mathrm{f}\right.$ $\left(C_{t-1}, S_{t}, P_{t}, H_{t}\right)$ than in model $3\left(C_{t 3}=\mathrm{f}\right.$ $\left(C_{t-1},\left(\frac{\mathrm{GDP}}{\text { Capita }}\right)_{t},\left(\mathrm{GDP}_{g}\right)_{t},\left(\mathrm{GDP}_{\text {tert }}\right)_{t}\right)$. Graphs illustrating the simulation of consumption $C_{t}$, and its residues are in Figure 3-i. Graphs illustrating the stability of the model are in Figure 4.

\subsection{Model CooB - Douglas}

The application of the logarithm on CooB - Douglass function permits us to have a linear form of the function.

In order to verify the absence of autocorrelation of residuals, we performed the Breusch-Godfrey test (BG) [20] on models 1, 2, 3 and 4, because in these models we have lag endogenous variables $\left(C_{t-1}\right)$ among exogenous variables. The Durbin-Watson test (DW) [34] shall be solely applied for models 5, 6 and 7 because there are no endogenous variables in the model.

Residuals are correlated at threshold $\alpha=5 \%$ if P-value $<$ $5 \%$. BG analysis test at order 4 and threshold $\alpha=5 \%$, shows that $\mathrm{P}$-value $>0.05$ for model 1,3 and 4 . $\mathrm{P}$-value $=$ $0.0427<0.05$ for model 2. meanwhile, the analysis of square residue correlograms permits us to say that there is no autocorrelation of residues, however, the critical probability of Ljung-Box Statistics (L-B) [34] is for all delays superior at threshold $\alpha=5 \%$. We can therefore accept the hypothesis of an absence of autocorrelation between residues for model 1, 2, 3 and 4. The Analysis of the squared residue correlograms, White's test (1980) and ARCH LM's test [15] applied to these four models permits us to accept the hypothesis of an absence of heteroscedasticity errors given that at the threshold $\alpha=5 \%$, P-value $>0.05$.

Our residue being homoscedastic it is thus white noise. DW test permits us to accept the hypothesis of an autocorrelation of residues, as models 5, 6 and 7 this test return values that are below 1.96 which is the critical value at the threshold $\alpha=5 \%$. For these models the autocorrelation can be rectified by introducing an autoregressive term. The results of these tests are listed in Table 4.

In order to verify if our white noise is Gaussian, we have 
performed Jarque -Bera's test (JB) [34]. The hypothesis of obtaining a Gaussian white noise is true at threshold $\alpha=$ $5 \%$, if Jarque -Bera's statistic is greater than that read from chi-square's table (JB stat $<5.99$ ) and the probability of Jarque -Bera's statistic provided by Eviews is superior to the provided threshold ( $\mathrm{P}$-value $>5 \%$ ).

The analysis of this test permits us to reject the hypothesis of a Gaussian white noise for all models except for Model 2. This model fulfill the conditions of Gaussian white noise namely $(\mathrm{JB}$ stat $=5.97<599)$ and $(\mathrm{P}$-value $=$ $0.0504>0.05)$. The CUSUM test [9] permits us to affirm the stability of our different models given that the curves are within the confident range at the threshold $\alpha=5 \%$. The results of the various tests are listed in Table 4.

For linear model, we have the following graphs: (A) forecast series in blue and forecast intervals to $95 \%$ in red. (B) Observation series in red, forecast series in green and residue in blue);
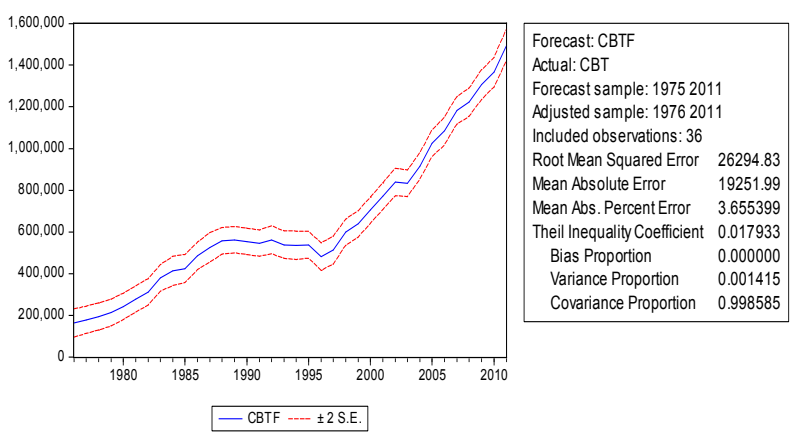

(A)

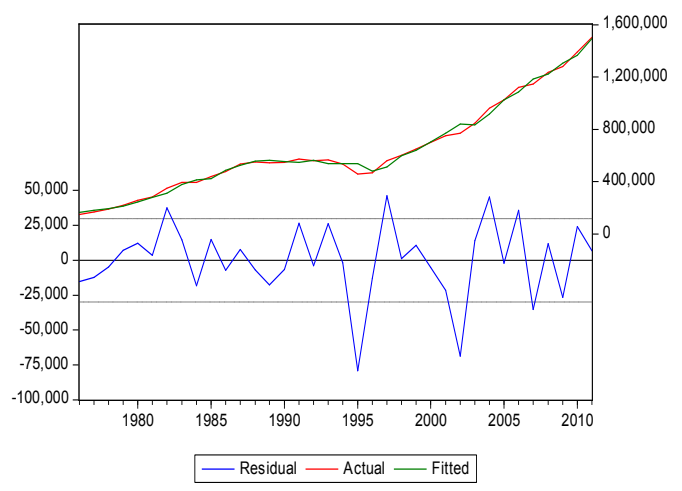

(B)

Figure 4. model 1

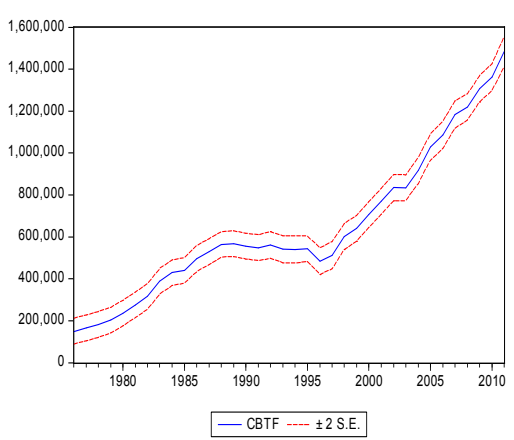

\section{Forecast: CBT} Actual: CBT Forecast sample: 1975201 Adjusted sample: 1976201 Included observations: 30 Root Mean Squared Error 27205.96 Mean Absolute Error $\quad 19992.92$ Mean Abs. Percent Error 3.567396 Theil Inequality Coefficient 0.018555 Bias Proportion $\quad 0.000000$ Variance Proportion $\quad 0.001515$ Covariance Proportion $\quad 0.998485$

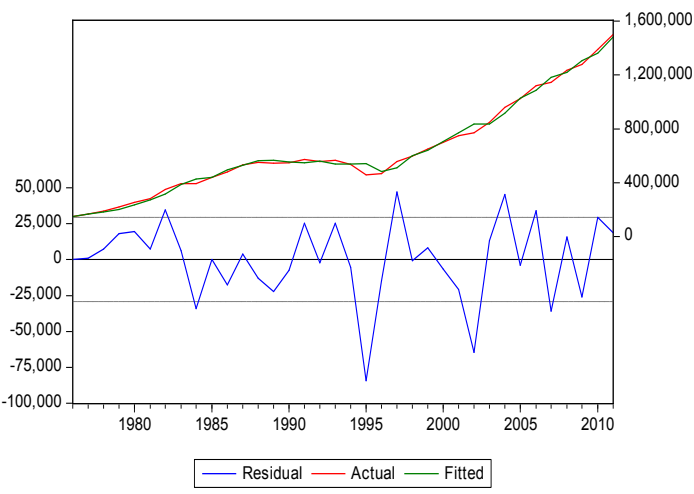

(B)

Figure 5. model 2
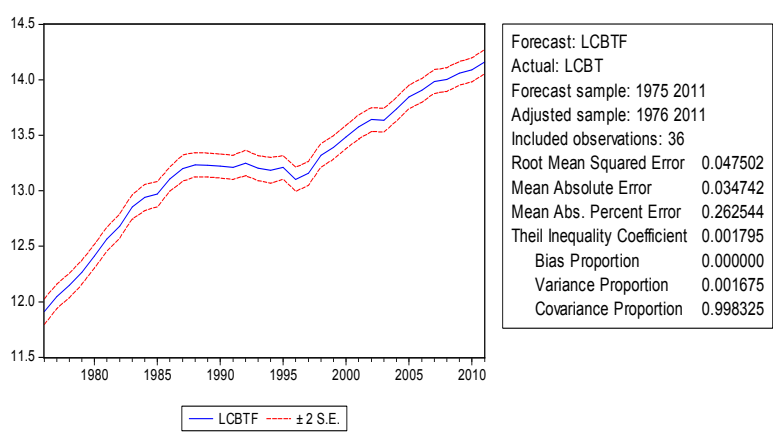

(A)

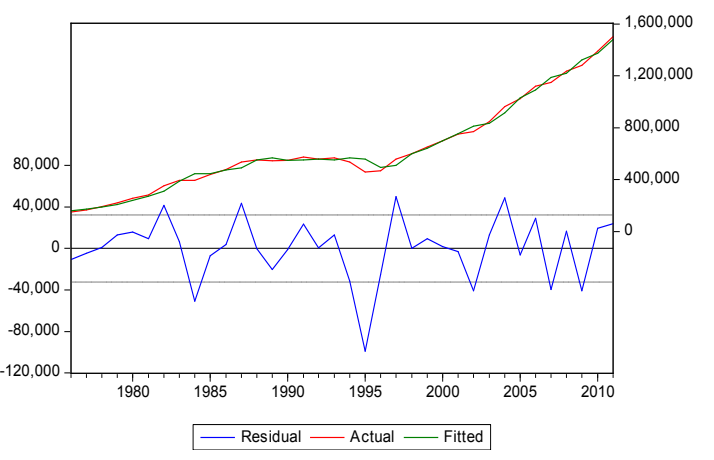

(B)

Figure 6. model 3
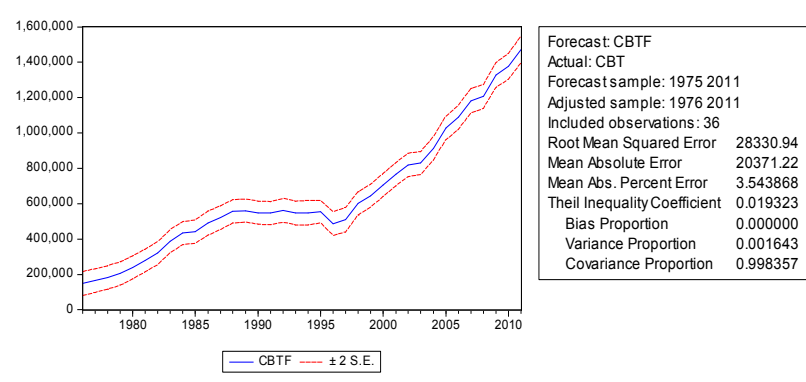

(A) 


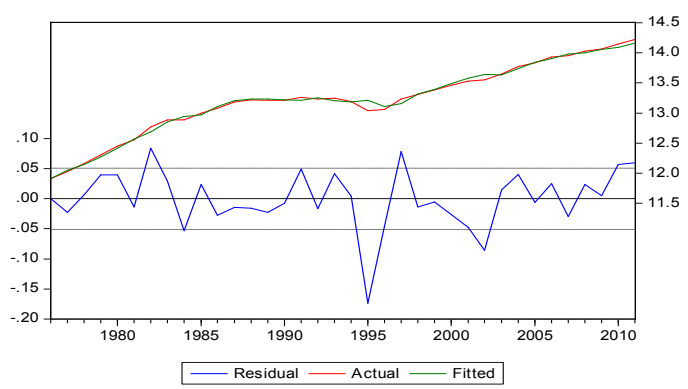

(B)

Figure 7. model 4

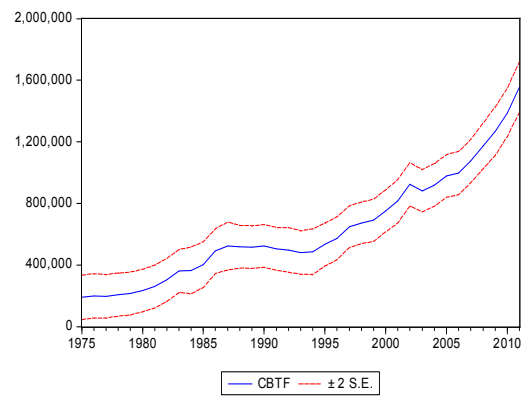

$$
\begin{array}{|ll|}
\hline \text { Forecast: CBTF } \\
\text { Actual: CBT } \\
\text { Forecast sample: } 19752011 \\
\text { Included observations: } 37 \\
\text { Root Mean Squared Error } & 58983.35 \\
\text { Mean Absolute Error } & 49319.84 \\
\text { Mean Abs. Percent Error } & 10.27457 \\
\text { Theil Inequality Coefficient } & 0.040819 \\
\hline \text { Bias Proportion } & 0.000000 \\
\text { Variance Proportion } & 0.006991 \\
\text { Covariance Proportion } & 0.993009 \\
\hline
\end{array}
$$

(A)

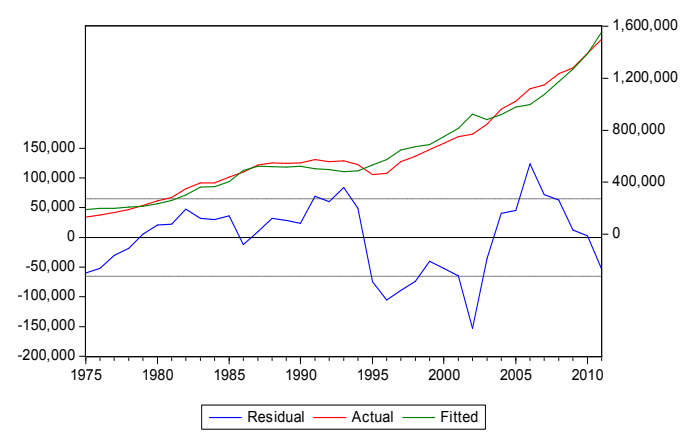

(B)

Figure 8.model 5

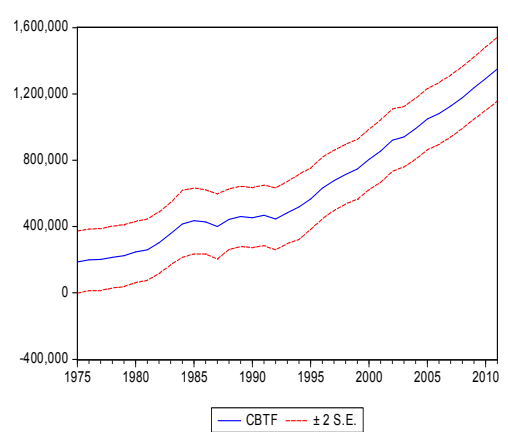

Forecast: CBTF Actual: CBT

Forecast sample: 1975201 ncluded observations: 37

Root Mean Squared Error 83969.86 Mean Absolute Error $\quad 70752.95$ Mean Abs. Percent Error 13.68953 Theil Inequality Coefficient 0.058211 Bias Proportion $\quad 0.000000$ $\begin{array}{ll}\text { Variance Proportion } & 0.014376 \\ \text { Covriance Proportion } & 0.985624\end{array}$

(A)

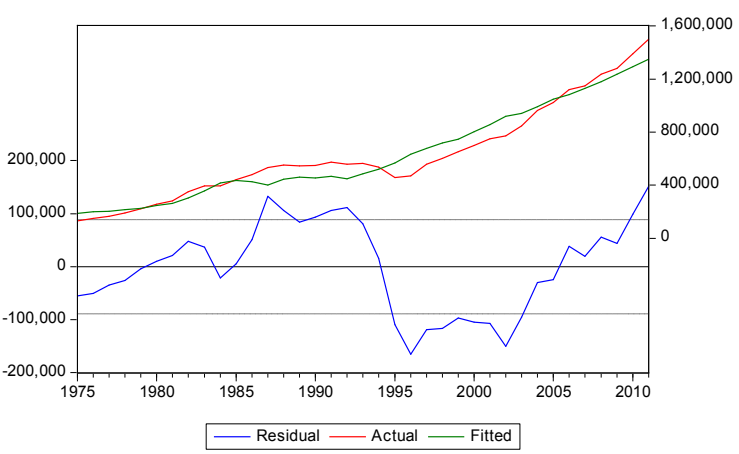

(B)

Figure 9.model 6

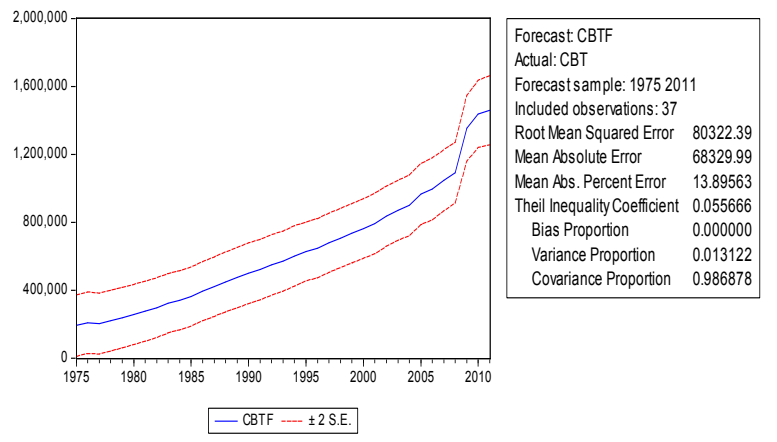

(A)

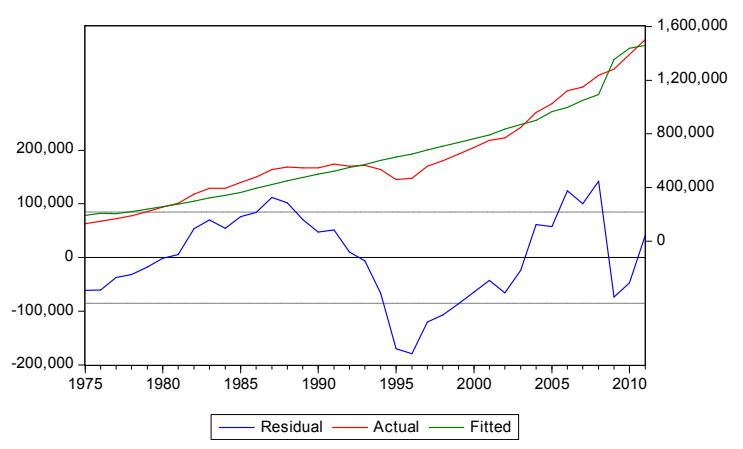

(B)

Figure 10. model 7

From the analysis of the different models, we can conclude that macro-economic indicators have a better influence on consumption than demographic indicators because SC and AIC are higher in Model $4\left(C_{t 4}=\mathrm{f}\right.$ $\left(C_{t-1}, S_{t}, P_{t}, H_{t}\right)$ than in model $3\left(C_{t 3}=\mathrm{f}\left(C_{t-1},\left(\frac{\mathrm{GDP}}{\text { Capita }}\right)_{t}\right.\right.$, $\left.\left(\mathrm{GDP}_{g}\right)_{t},\left(\mathrm{GDP}_{\text {tert }}\right)_{t}\right)$. Graphs illustrating the simulation of consumption $C_{t}$, and its residues are in Figure 3-i. Graphs illustrating the stability of the model are in Figure 4.

\subsection{Model CooB - Douglas}

The application of the logarithm on CooB - Douglass function permits us to have a linear form of the function. 
Table 5. Regression coefficients for Coob-Douglass models

\begin{tabular}{|c|c|c|c|c|c|c|c|c|}
\hline \multirow{2}{*}{ Explanation } & \multicolumn{2}{|l|}{ Model 1' } & \multicolumn{2}{|l|}{ Model 2' } & \multicolumn{2}{|l|}{ Model 3' } & \multicolumn{2}{|l|}{ Model 4' } \\
\hline & Coef. & Prob.(p-value) & Coef. & Prob.(p-value) & Coef. & Prob.(p-value) & Coef. & Prob.(p-value) \\
\hline \multirow{2}{*}{$C_{t-1}$} & 0.8396 & 0 & 0.8698 & 0 & \multirow{4}{*}{$0.8193(16.550)$} & 0 & 0.9988 & 0 \\
\hline & -10.12 & $0.0829 *$ & -12.3945 & $0.0701 *$ & & $0.049504 *$ & -11.9077 & $0.0838^{*}$ \\
\hline \multirow{2}{*}{$(\mathrm{S})_{\mathrm{t}}$} & -0.0213 & 0.843 & -0.0973 & 0.0991 & & & -0.2076 & 0.0781 \\
\hline & $(-0.199)$ & $0.1067^{*}$ & $(-1.6985)$ & $0.0573 *$ & & & $(-1.8218)$ & $0.1139 *$ \\
\hline \multirow{2}{*}{$\mathrm{H}_{\mathrm{t}}$} & 0.3078 & 0.3158 & & & & & $-0.0097(-$ & 0.9778 \\
\hline & (1.0207) & $0.3015^{*}$ & & & & & $0.0280)$ & $0.3462 *$ \\
\hline \multirow{2}{*}{$P_{t}$} & -0.2929 & 0.5353 & & & & & 0.422 & 0.4072 \\
\hline & $(-0.627)$ & $0.4668^{*}$ & & & & & -0.8401 & $0.5023^{*}$ \\
\hline GDP & & & & & 0.0578 & 0.7416 & & \\
\hline$(\overline{\text { Capita }})_{\mathrm{t}}$ & & & & & -0.3326 & 0.1737 & & \\
\hline \multirow{2}{*}{$\mathrm{GDP})_{\mathrm{t}}$} & 0.4948 & 0.0011 & 0.2554 & 0.0001 & \multirow{2}{*}{$0.4166(1.7306)$} & 0.0935 & & \\
\hline & 3.6082 & 0.1371 & -4.6016 & $0.0555^{*}$ & & $0.2407 *$ & & \\
\hline \multirow{2}{*}{$\left(\mathrm{GDP}_{\text {tert }}\right)_{\mathrm{t}}$} & -0.3748 & 0.0767 & & & -0.2831 & 0.156 & & \\
\hline & $(-1.835)$ & $0.2042 *$ & & & $(-1.453)$ & $0.1947 *$ & & \\
\hline \multirow{2}{*}{$\alpha_{0}$} & 1.4076 & 0.6744 & 0.86 & 0.0003 & \multirow{2}{*}{$0.7495(1.5779)$} & 0.1247 & -4.0704 & 0.1741 \\
\hline & -0.4244 & $3.3165^{*}$ & -4.0984 & $0.2098^{*}$ & & $0.4750^{*}$ & $(-1.3910)$ & $2.9262 *$ \\
\hline
\end{tabular}

Table 5. continued

\begin{tabular}{|c|c|c|c|c|c|c|}
\hline \multirow{2}{*}{ Explanation } & \multicolumn{2}{|l|}{ Model 5, } & \multicolumn{2}{|l|}{ Model 6' } & \multicolumn{2}{|l|}{ Model 7' } \\
\hline & Coef. & Prob.(p-value) & Coef. & Prob.(p-value) & Coef. & Prob.(p-value) \\
\hline \multicolumn{7}{|l|}{$\mathrm{C}_{\mathrm{t}-1}$} \\
\hline \multirow{2}{*}{$(\mathrm{S})_{\mathrm{t}}$} & 0.8621 & 0 & & & 0.852733 & 0 \\
\hline & -7.0239 & $0.1227^{*}$ & & & -5.1341 & $0.1660^{*}$ \\
\hline \multirow{2}{*}{$\mathrm{H}_{\mathrm{t}}$} & 1.7159 & 0.004 & & & 1.937383 & 0.0094 \\
\hline & -3.112 & $0.5513^{*}$ & & & -2.7577 & $0.7025^{*}$ \\
\hline \multirow{2}{*}{$\mathrm{P}_{\mathrm{t}}$} & -2.9233 & 0.0009 & & & -2.0803 & 0.0581 \\
\hline & $(-3.653)$ & $0.8002 *$ & & & $(-1.9628)$ & $1.0598^{*}$ \\
\hline \multirow{2}{*}{$\left(\frac{\mathrm{GDP}}{\text { Capita }}\right)_{\mathrm{t}}$} & & & -1.0595 & 0.0501 & & \\
\hline & & & $(-2.033)$ & 0.521 & & \\
\hline \multirow{2}{*}{$\mathrm{GDP})_{\mathrm{t}}$} & 0.9422 & 0.0012 & 1.9535 & 0.0105 & & \\
\hline & -3.5638 & $0.2643 *$ & 2.715 & 0.7194 & & \\
\hline \multirow{2}{*}{$\left(\mathrm{GDP}_{\text {tert }}\right)_{\mathrm{t}}$} & -0.3533 & 0.4038 & -0.3048 & 0.6227 & & \\
\hline & $(-0.846)$ & $0.4174 *$ & $(-0.496)$ & 0.6136 & & \\
\hline \multirow{2}{*}{$\alpha_{0}$} & 19.8069 & 0.0015 & 5.2782 & 0.0002 & 8.104594 & 0.2093 \\
\hline & -3.4745 & $5.7006^{*}$ & -4.2036 & $1.2556^{*}$ & -1.2804 & $6.3297 *$ \\
\hline
\end{tabular}

() t-statistic, *adjs. standard error, _ the related variable is not significant

The ADF and PP test performed at the variables after application of the logarithm permits us to observe that our variables are stationary at different orders: series $\ln \left(S_{t}\right)$ and $\ln \left(P_{t}\right)$ are stationary at zero-order and other series are integrated of order 1 . Results of these tests are included in Table 2. Student's test permits us to identify the best model has been that which dependent on $\ln \left(S_{t}\right),\left(\ln \left(\mathrm{GDP}_{g}\right)_{t}\right)$ and (ln $\left(C_{t-1}\right)$. It corresponds to model 2'. Fischer test permits us to observe that in models 5 and 7 the coefficients are not globally significant. The results of regression coefficients for linear models are listed in Table 5.

BD and LB tests performed on residues of models 1, 2, 3 and 4 accept the hypothesis of an absence of an autocorrelation of residuals for these models. DW test performed on residues of models 5, 6 and 7 refutes the hypothesis of an absence of autocorrelation of residues. Residues are thus correlated for these three models. The Analysis of the squared correlogram residues, $\mathrm{ARCH}$ and White tests applied to models 1, 2, 3 and 4 permit to conclude that the residues are homoskedastic (white noise). JB test refutes the hypothesis of Gaussian white noise for all the different models. CUSUM test affirms the stability of our different models except for Model 6', the reason being that its curve is not within the range of the confident threshold $\alpha=5 \%$. Results of various tests for the models are listed in Table 6. 
Table 6. Regression coefficients for Coob-Douglas models

\begin{tabular}{|c|c|c|c|c|c|c|c|c|c|c|c|c|}
\hline \multirow{2}{*}{$\begin{array}{l}\text { Coob- } \\
\text { Douglass } \\
\text { Models }\end{array}$} & \multirow{2}{*}{$\mathbf{R}^{2}$} & \multirow{2}{*}{$\mathbf{R}^{2}$} & \multirow{2}{*}{ SE Reg. } & \multirow{2}{*}{$\begin{array}{l}\text { Prob. } \\
\text { (F-Stat) }\end{array}$} & \multirow{2}{*}{ AIC } & \multirow{2}{*}{ SC } & \multicolumn{2}{|c|}{ White -test } & \multirow{2}{*}{ JB-test } & \multirow{2}{*}{ F -Stat } & \multicolumn{2}{|l|}{$\begin{array}{l}\text { B-D (LM) } \\
\text { D-W }\end{array}$} \\
\hline & & & & & & & $\begin{array}{l}\text { ORS } \\
\text { (p-value) }\end{array}$ & $\begin{array}{l}\text { F-stat } \\
\text { (p-value) }\end{array}$ & & & $\begin{array}{l}\text { LM } \\
\text { (p-value) }\end{array}$ & $\begin{array}{l}\text { F- Stat } \\
\text { (p-value) }\end{array}$ \\
\hline Model 1' $\left(\boldsymbol{C}_{\boldsymbol{t 1}}{ }^{\prime}\right)$ & 0.9937 & 0.9925 & 0.0509 & 0.0000 & -2.94 & -2.6334 & $\begin{array}{l}20.1681 \\
(0.3234)\end{array}$ & $\begin{array}{l}1.2031 \\
(0.3535)\end{array}$ & $\begin{array}{l}26.1531 \\
\left(2 *^{*} 10^{\mathrm{E}}-6\right)\end{array}$ & 774.5725 & $\begin{array}{l}10.9971 \\
(0.0266)\end{array}$ & $\begin{array}{l}2.7489 \\
(0.050)\end{array}$ \\
\hline Model 2' $\left(\boldsymbol{C}_{\boldsymbol{t} 2}{ }^{\prime}\right)$ & 0.9929 & 0.9922 & 0.0517 & 0.0000 & -2.98 & -2.8050 & $\begin{array}{l}8.8248 \\
(0.4536)\end{array}$ & $\begin{array}{l}0.9381 \\
(0.5099)\end{array}$ & $\begin{array}{l}21.3625 \\
\left(2.3 * 10^{\mathrm{E}}-4\right)\end{array}$ & 1504.179 & $\begin{array}{l}9.8214 \\
(0.0435)\end{array}$ & $\begin{array}{l}2.6261 \\
(0.0557)\end{array}$ \\
\hline Model 3' $\left(\boldsymbol{C}_{\boldsymbol{t} 3}{ }^{\prime}\right)$ & 0.9933 & 0.9924 & 0.0511 & 0.0000 & -2.97 & -2.7583 & $\begin{array}{l}16.070 \\
(0.3091)\end{array}$ & $\begin{array}{l}1.2095 \\
(0.3375)\end{array}$ & $\begin{array}{l}21.6423 \\
\left(2 *^{*} 10^{\mathrm{E}}-5\right)\end{array}$ & 1152.618 & $\begin{array}{l}5.5030 \\
(0.2395)\end{array}$ & $\begin{array}{l}1.2180 \\
(0.3263)\end{array}$ \\
\hline Model 4' $\left(\boldsymbol{C}_{\boldsymbol{t} \boldsymbol{4}}{ }^{\prime}\right)$ & 0.9903 & 0.9890 & 0.0615 & 0.0000 & -2.60 & -2.38 & $\begin{array}{l}7.9173 \\
(0.4416)\end{array}$ & $\begin{array}{l}0.9515 \\
(0.4926)\end{array}$ & $\begin{array}{l}11.2780 \\
(0.0035)\end{array}$ & 1504.179 & $\begin{array}{l}5.2807 \\
0.2597\end{array}$ & $\begin{array}{l}1.1603 \\
(0.3501)\end{array}$ \\
\hline $\begin{array}{l}\text { Model 5' } \\
\left(\boldsymbol{C}_{t 5}\right)\end{array}$ & 0.9757 & 0.9718 & 0.1052 & 0.0000 & -1.51 & -1.256 & & & & 249.8790 & {$[0.6583]$} & \\
\hline $\begin{array}{l}\text { Model 6' } \\
\left(\boldsymbol{C}_{t 6}\right)\end{array}$ & 0.9367 & 0.9310 & 0.16480 & 0.0000 & -0.66 & -0.4922 & & & & 162.9722 & [0.2009] & \\
\hline $\begin{array}{l}\text { Model } 7 \\
\left(\boldsymbol{C}_{t 7}\right)\end{array}$ & 0.9526 & 0.9483 & 0.1425 & 0.0000 & -0.95 & -0.7817 & & & & 221.3877 & {$[0.2976]$} & \\
\hline
\end{tabular}

() p-value, [ ]Durbin-Watson-stat.

The result of regression coefficients for Coob-Douglas models are listed in Table 5. Graphs illustrating the simulation of low voltage consumption and their residues are in Figure 11 to 17. Graphs illustrating the stability of the model are in Figure 18.

From the analysis of different models, we can conclude that the macro-economic indicators have a greater impact on consumption than demographic indicators because SC and AIC are higher in model $4^{\prime}\left(C_{t 4^{\prime}}=\mathrm{f}\left(\ln \left(C_{t-1}\right), \ln \left(S_{t}\right)\right.\right.$, $\left.\ln \left(P_{t}\right), \ln \left(H_{t}\right)\right)$ in the model $3^{\prime}\left(C_{t 3^{\prime}}=\mathrm{f}\left(\ln \left(C_{t-1}\right)\right.\right.$, $\ln \left(\left(\frac{\mathrm{GDP}}{\text { Capita }}\right)_{t}\right), \ln \left(\left(\mathrm{GDP}_{g}\right)_{t}\right), \ln \left(\left(\mathrm{GDP}_{\text {tert }}\right)_{t}\right)$.

Conclusively, we can assert that for CooB-Douglass' model, the determining coefficient $\mathrm{R}^{2}$ is between 0.9310 and 0.9925 , AIC is between -0.66 and $-2.95, \mathrm{SC}$ is between -0.49 and -2.8 . For the linear model, $\mathrm{R}^{2}$ is between 0.9390 and 0.9931 , AIC is between 23.6990 and 25.7305 , SC is between 23.6990 and 25.9046. AIC and SC been minimum in CooB-Douglass model, we can thus conclude that, this model generally provide better results than the linear model, given that the application of the logarithm on these different variables permits to captures the Heteroscedasticity that could be observe

For Coob-Douglas models, we have the following graphs: (A) forecast series in blue and forecast intervals at $95 \%$ in red. (B) Observation series in red, forecast series in green and residue in blue),

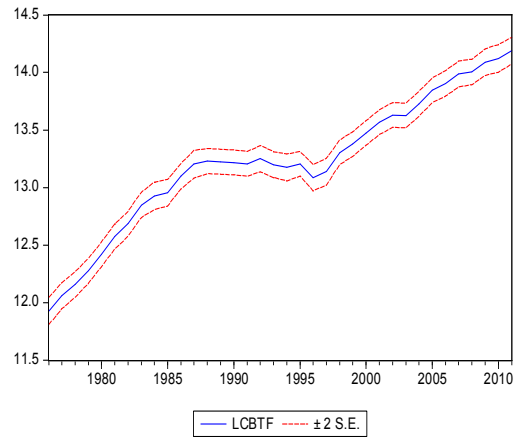

(A)

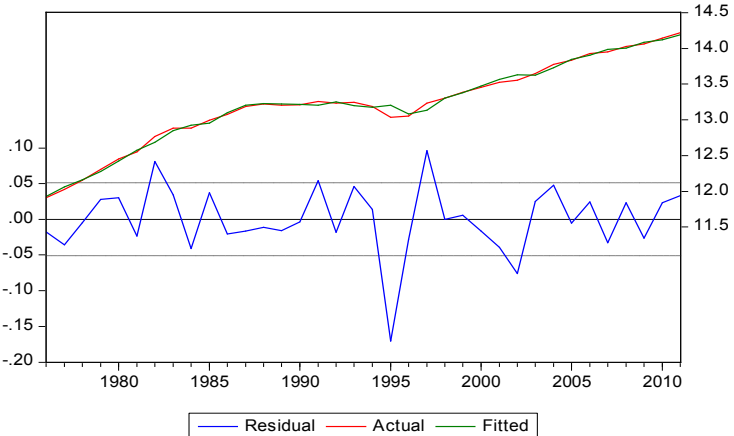

(B)

Figure 11. model 1'

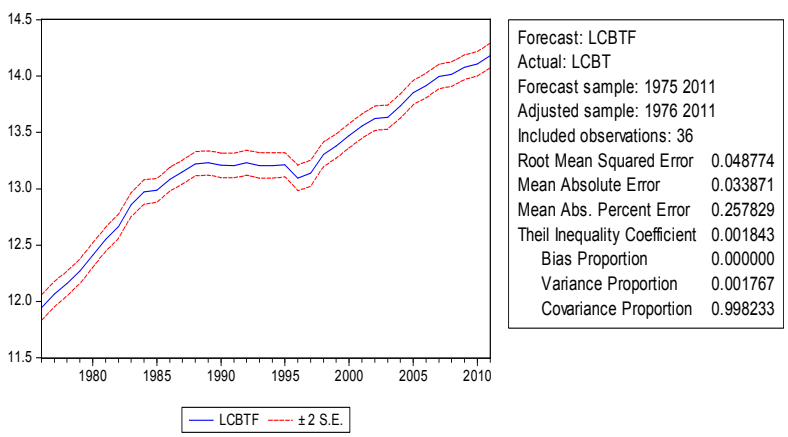

(A)

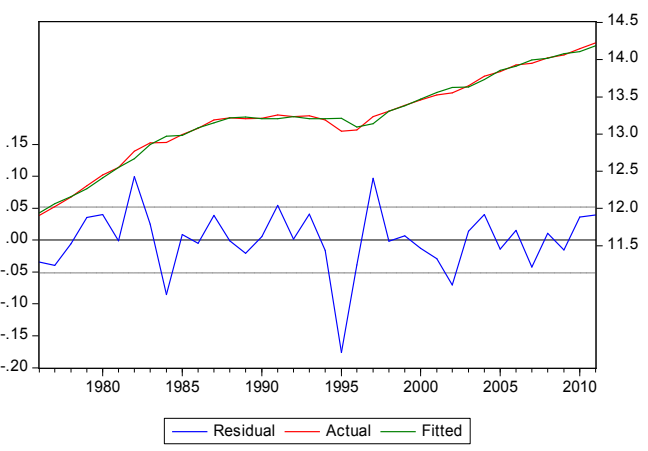

(B)

Figure 12. model 2' 


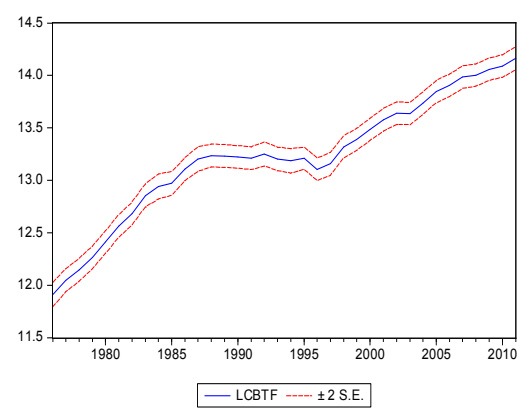

(A)

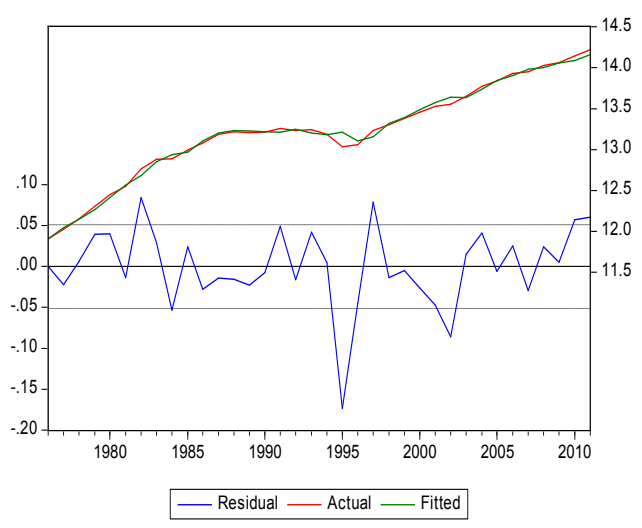

(B)

Figure 13. model 3'

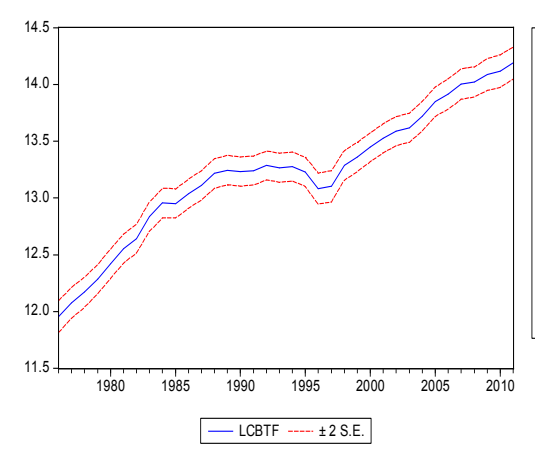

(A)

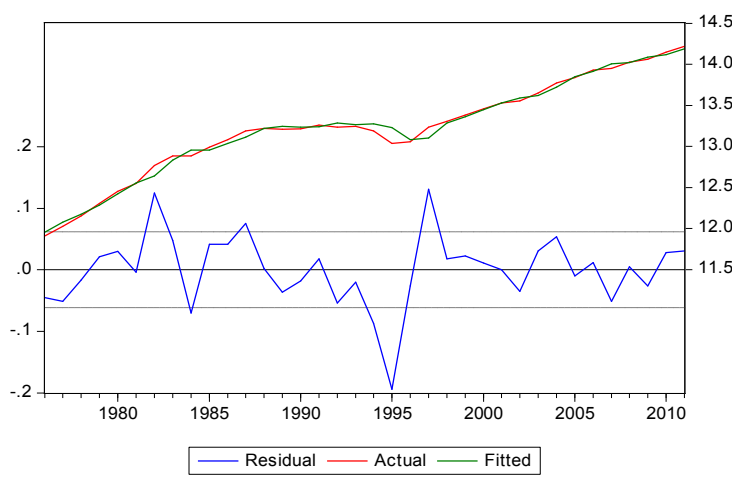

(B)

Figure 14. model 4,
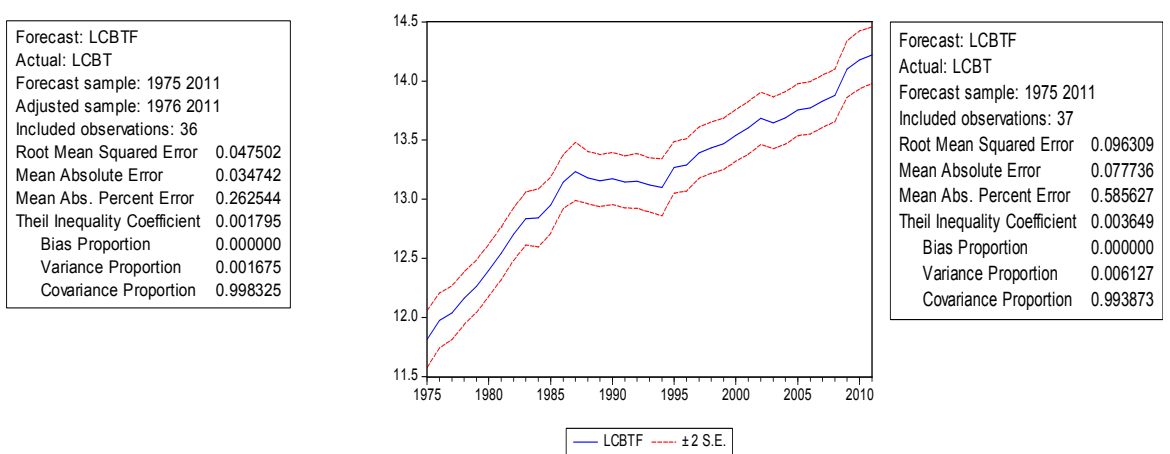

(A)

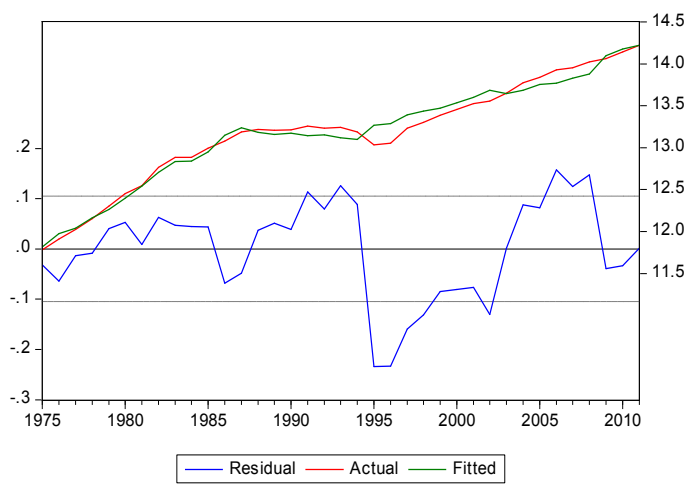

(B)

Figure 15. model 5,
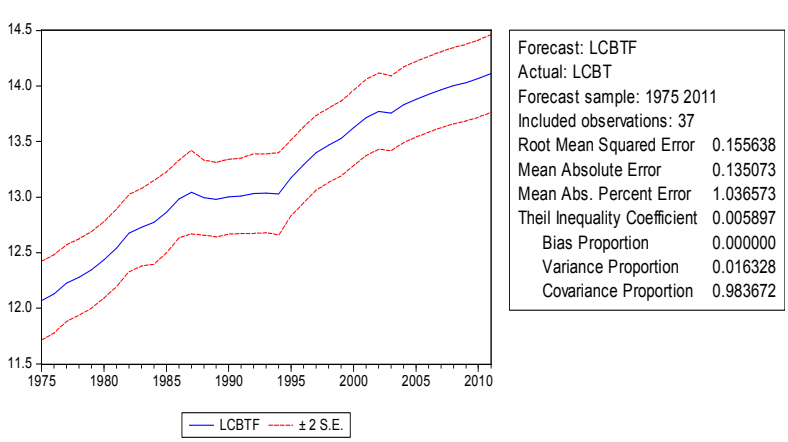

(A)

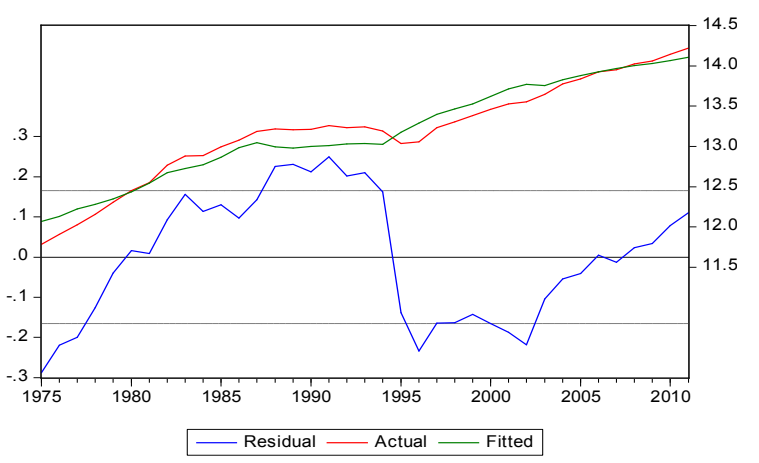

(B)

Figure 16. model 6' 

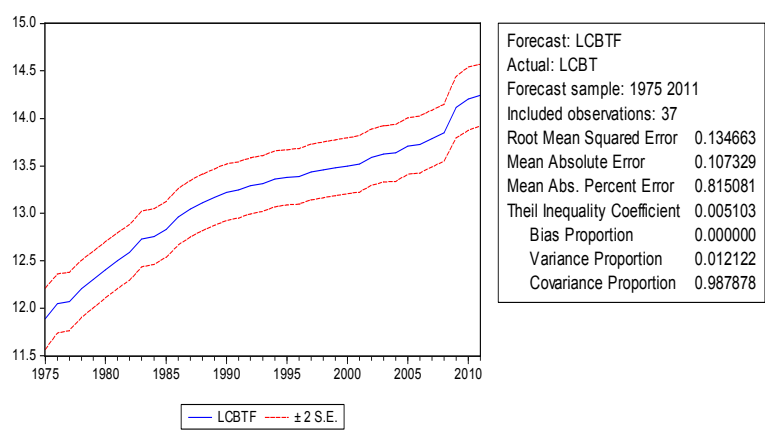

(A)

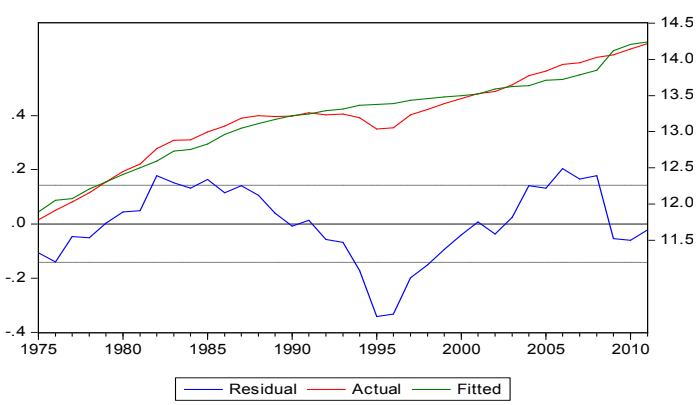

(B)

Figure 17. model 7

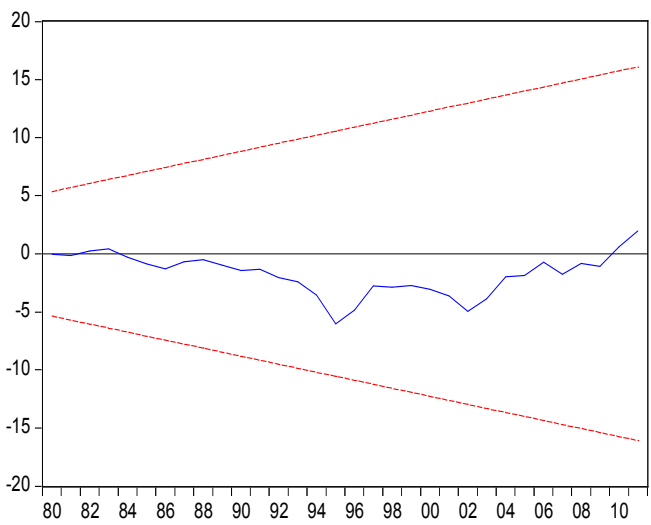

- CUSUM --. $5 \%$ Significance

$\mathrm{C}_{\mathrm{t} 2}$

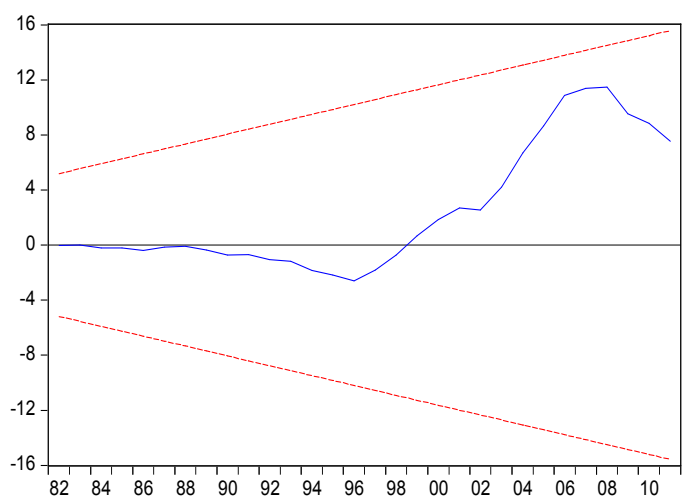

- CUSUM ---. $5 \%$ Significance

$\mathrm{C}_{\mathrm{t} 5}$

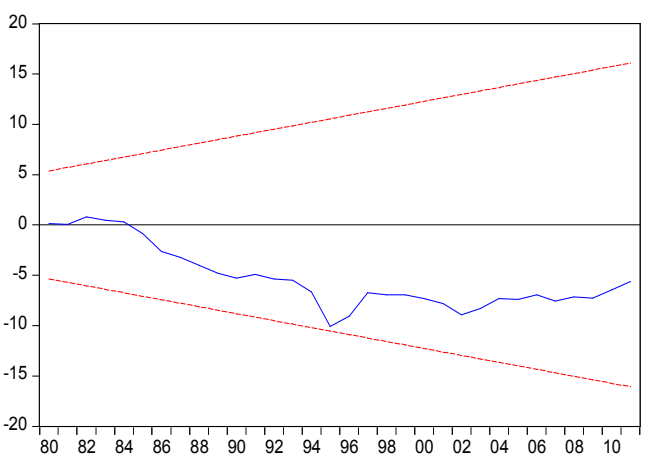

_ CUSUM ---- $5 \%$ Significance

$\mathrm{C}_{\mathrm{t} 2}$

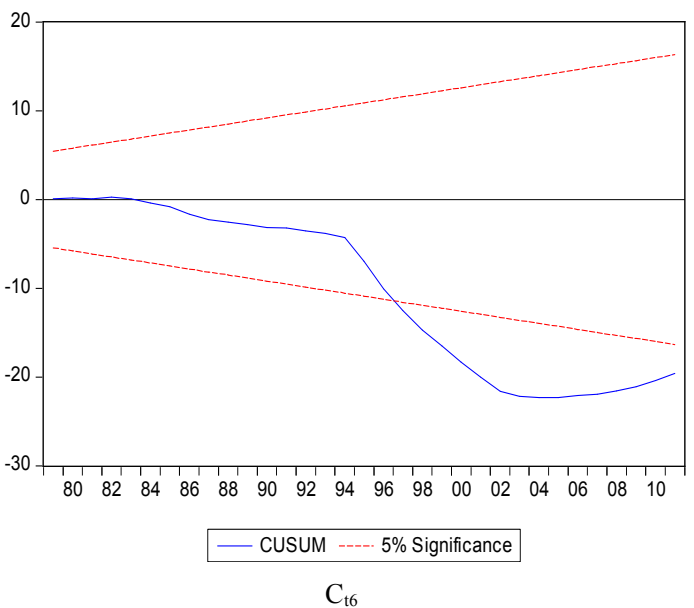

Figure 18. CUMUS test

\section{Conclusion and Discussion}

This article presents the modeling of electricity demand of Cameroonian Low Voltage customers using econometric techniques particularly the linear regression model and CooB-Douglass' model. The socio -economic indicators (demographic and macroeconomic) and past consumption are used as exogenous variables. It is sterms from this analysis that $\mathrm{CooB}$ - Douglass' models provide better results than linear models. The best linear model is that which includes $\left(\mathrm{GDP}_{g}\right)_{t}, P_{t}$ and $C_{t-1}$, and the best of CooBDouglass' model is that which includes $\left(\mathrm{GDP}_{g}\right)_{t}$ of $S_{t}$ and $C_{t-1}$, here, we have model 2 and 2' respectively because they have the smallest AIC and $\mathrm{SC}$ and their residues are white noise. At the outcome of this analysis, we find that demographic and macroeconomic indicators having a significant influence on consumption are respectively the population and $\left(\mathrm{GDP}_{g}\right)_{t}$ for the linear model and the number of subscribers $S_{t}$ and $\left(\mathrm{GDP}_{g}\right)_{t}$ for CooB-Douglass' model. We note in this model that the coefficient of $\left(\mathrm{GDP}_{g}\right)_{t}$ is more significant than that of the population. There after, we find that the absence of the past consumption variable in a model causes the autocorrelation of its residues as is the case in models 5, 6 and 7 of the linear model and 5' , $6^{\prime}$ and $7^{\prime}$ CooB-Douglass' model. 
Past consumption therefore influences consumption strongly because it indicates the presence of a dynamic adjustment of $C_{t}$ in these different models. Models 3, 4, 3 ' and 4 ' permit us to arrive at the conclusion that macro economic indicators have a better influence on consumer than demographic indicators. This might be due to the fact that approximately $71 \%$ of low voltage customers in Cameroon are households. Consumption been principally of a domestic nature, the observed historical data implicitly hold that, an individual is averagely predisposition to see his power consumption increase, depending on the amelioration of his living standards and the overall increasing trend of the volume of Low Voltage consumption based on population growth and therefore, the number of households and Low Voltage subscribers.

It would be beneficial for the country to invest in renewable energy for rural electrification or remote areas which do not have access to electricity as it is been done already in the Northern part of the country through the exploitation of the solar potential of the region. To overcome blackout and voltage drop, it would be important to conduct a household survey so as to identify deposits consumption especially at rush hour. It is also important to carry out an awareness campaign so as to convince the people to use low power consuming devices that will help curb or reduce their electricity bills while improving the quality of electricity received.

Mastery of electricity demand (MDE) been a factor that can strongly influence the consumption of electricity, it will be interesting to run a scenario test on electricity consumption involving the MDE so as to evaluate the impact that it could have on Cameroonian low voltage consumers. The information presented in this article can help stake holders to identify the relationship that exist between the consumption of low voltage customers and different socio -economic indicators. Relying on this information, a better plan for the electricity network sector can be put in place and thus breach the gap between supply and demand Cameroon.

\section{Acknowledgements}

The authors thank Honore Lafon for his help in the translation. We would like to express our sincere gratitude to the anonymous reviewers and the Editor of the Journal for many valuable comments.

\section{References}

[1] AES - SONEL ,2011 Rapport AES/AREVA.

[2] Annuaire statistique 2010 du Cameroun - INS et Document de Stratégie pour la Croissance et l'Emploi- Rapport Définitif - Août 2009.

[3] Development Indicators' (1960 - 1994) et INS / Comptabilité Nationale $(1995-2009)$.
[4] Andrews, Donald W. K. (1993). "Tests for Parameter Instability and Structural Change with Unknown Change Point," Econometrica, 61(4), 821-856.R. Nicole, "Title of paper with only first word capitalized," J. Name Stand. Abbrev., in press.

[5] Bernard, Jean-Thomas, "Un modèle Intégré de la Demande Totale d'Énergie : Application à la Province de Québec" Green, Université Laval, 2000.

[6] Bernard, Jean-Thomas et al., "Quebec Residential Electricity Demand: A Microeconomic Approach",The Canadian Journal of Economics, Vol 29, No 1,1996,92-113.

[7] Bollerslev, Tim, Robert F. Engle and Daniel B. Nelson (1994). "ARCH Models," Chapter 49 in Robert F.

[8] Breusch, T. S., and A. R. Pagan (1979). "A Simple Test for Heteroskedasticity and Random Coefficient Variation," Econometrica, 48, 1287-1294.

[9] Brown, R. L., J. Durbin, and J. M. Evans (1975). "Techniques for Testing the Constancy of Regression

[10] Comptabilité Nationale - Annuaires Statistiques INS, MINFI pour PIB nominal et PIB réel (à prix constants).

[11] C. Adjamagbo, P. Ngae, A. Vianou, V. Vignero « Modélisation de la demande en énergie électrique au Togo », Revue des Energies Renouvelables Vol. 14 N$^{\circ} 1$ (2011) 67 83.

[12] Dubin, Jeffrey A., McFadden,Daniel L., "An Econometric Analysis of Residential Electric Appliance Holdings and Consumption ",Econometrica, Vol 52,No2,1984,345-362.

[13] Doornik J.A. et H. Hansen (1994): « A Practical Test for Univariate and Multivariate Normality ", Discussion paper, Nuffield College.

[14] Dickey, D.A., Fuller, W.A., (1981) Likelihood ratio statistics for autoregressive time series with a unit root. Econometrica $49,1057-1072$.

[15] Engle, R.F., Granger, C.W.J., (1987) Co-integration and error correction: representation estimation and testin. Econometrica 55, 251-276.

[16] Engle, Robert F. and K. F. Kroner (1995). "Multivariate Simultaneous Generalized ARCH," Econometric Theory, 11, 122-150.

[17] Esseghir Asma et Haouaoui Leila. (2011) « Croissance économique, consommation d'énergie et développement durable : l'exemple de la région méditerranéenne ». Colloque international francophone, "Le développement durable : débats et controverses », 15 et 16 décembre 2011, Université Blaise Pascal, Clermont-Ferrand.

[18] EViews. http://www.eviews.com

[19] Fair, Ray C. (1970). "The Estimation of Simultaneous Equation Models with Lagged Endogenous Variables and First Order Serially Correlated Errors," Econometrica, 38, 507-516.

[20] Godfrey, L. G. (1988).Specification Tests in Econometrics, Cambridge: Cambridge University Press.

[21] Godfrey, L. G. (1978). "Testing for Multiplicative Heteroscedasticity," Journal of Econometrics, 8, 227-236. 
[22] Godfrey L.G. (1978): « Testing for Higher Order Serial Correlation in Regression Equations when the Regressors Include Lagged Dependent Variables », Econometrica, 46, pp. 1303-1313.

[23] Gaetan Lafrance , «Mathématiques et modélisation énergétique Intégration de l'éolien et prévision de demande dans un réseau électrique » Bulletin AMQ, Vol. XLVII, no3, octobre $2007-68$ Actes du 50e congrès.

[24] Guertin, Chantal, et al, "Determining Demand for EnergyServices: Investigating income-driven behaviours", State University of New York at Binghamton, 2003.

[25] Hausman, Jerry A., Joskow, Paul 1., "Evaluating the Cost and Benefits of Appliance Efficiency Standards",The American Economic Review, Vol 72, No2, 1982.

[26] Issa SACKO (2004) « Analyse Des Liens Entre Croissance Economique Et Consommation D'énergie Au Mali ». MSAS'.

[27] Judge, George G., W. E. Griffiths, R. Carter Hill, Helmut Lütkepohl, and Tsoung-Chao Lee (1985). The Theory and Practice of Econometrics, 2nd edition, New York: John Wiley \& Sons.

[28] Krolzig H.-M. ET D.F. Hendry (2001): " Computer Automation of General-to-Specific Model Selection Procedures », Journal of Economic Dynamics and Control, 25 (6-7), pp. 831-866.Nicholls D.F. et A.R.

[29] Pagan (1983): « Heteroscedastici ty in Models with Lagged Dependent Variables », Econometrica, n51, pp.1233-1242.

[30] Patrice Ongono "Energy consumption and economic performance in Cameroon" MPRA Paper No. 23525, posted 27. June 2010 10:13 UTC.
[31] Reiss, Peter C., White, Matthew W.,"Household Electricity Demand", Stanford University, 2004.

[32] Samuel AMBAPOUR, Christophe MASSAMBA (2005). « Croissance économique et consommation d'énergie au Congo : une analyse en termes de causalité ». DT 12/2005.

[33] SIE-Cameroon, 2010. Cameroon energy information system: Report 2010. Ministry of Energy and Water resources.

[34] R. Starts, EViews Illustrated for Version 7.2, 1st Ed., (2012) Micro Software, LLC, 2012.

[35] White, Halbert (1980)."A Heteroskedasticity-Consistent Covariance Matrix and a Direct Test for Heteroskedasticity," Econometrica, 48, 817-838.

[36] Yusri Syam Akil, Hajime Miyauchi. (2013) Seasonal Peak Electricity Demand Characteristics: Japan Case Study. International Journal of Energy and Power Engineering. Vol. 2, No. 3, 2013, pp. 136-142. doi: $10.11648 /$ j.ijepe.20130203.18.

[37] TIMITE E. H. Hassane , Dr.AHOURE Alban.(2010) Consommation d'Électricité et Croissance Économique en Côte d'Ivoire.

[38] Engle and Daniel L. McFadden (eds.), Handbook of Econometrics, Volume 4, Amsterdam: Elsevier Science B.V.

[39] Yris D. Fondja Wandji, (2013) Energy Policy, Energy consumption and economic growth: Evidence from Cameroon, Vol. 61 1295-1304 